30

\title{
Micro-topographical guidance of macropinocytic signaling patches
}

(1)

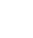

$$
\text { Gen Honda }{ }^{1} \text {, Nen Saito }{ }^{3,4} \text {, Taihei Fujimori }{ }^{1} \text {, Hidenori Hashimura }{ }^{1} \text {, Mitsuru J. Nakamura }{ }^{1} \text {, }
$$$$
\text { Akihiko Nakajima }{ }^{1,2} \text {, Satoshi Sawai }{ }^{* 1,2,3,5}
$$

\footnotetext{
${ }^{1}$ Department of Basic Science, Graduate School of Arts and Sciences, University of Tokyo, 3-8-1
}

Komaba, Meguro-ku, Tokyo 153-8902, Japan.

${ }^{2}$ Research Center for Complex Systems Biology, University of Tokyo, 3-8-1 Komaba, Meguro-ku, Tokyo 153-8902, Japan.

${ }^{3}$ Universal Biological Institute, University of Tokyo, Bunkyo-ku, Tokyo 113-0033, Japan

${ }^{4}$ Exploratory Research Center on Life and Living Systems, National Institutes of Natural Sciences, Okazaki, Aichi 444-8787, Japan

${ }^{5}$ Department of Biology, Graduate School of Science, University of Tokyo, 7-3-1 Hongo, Bunkyoku, Tokyo 113-0033, Japan.

*Corresponding author: cssawai@mail.ecc.u-tokyo.ac.jp

\section{Abstract}

In fast moving cells such as amoeba and immune cells, spatio-temporal regulation of dendritic actin filaments shapes large-scale plasma membrane protrusions. Despite the importance in migration as well as in particle and liquid ingestion, how these processes are affected by the micrometer-scale surface features is poorly understood. Here, through quantitative imaging analysis of Dictyostelium on micro-fabricated surfaces, we show that there is a distinct mode of topographically guided cell migration 'phagotaxis' directed by the macropinocytic Ras/PI3K signaling patches. The topography guidance was PI3K-dependent and involved nucleation of a patch at the convex curved surface and confinement at the concave surface. Due to the topography-dependence, constitutive cup formation for liquid uptake in the axenic strain is also destined to trace large surface features. Given the fact that PI3K-dependency of phagocytosis are restricted to large particles in both Dictyostelium and immune cells, topography-dependency and the dual-use of membrane cups may be wide-spread. 


\section{Introduction}

33 Large-scale deformation of plasma membrane during cell migration, particle and liquid ingestion 34 depends on physical cues such as substrate rigidity and topography (Champion and Mitragotri, 2006; 35 Clarke et al., 2010; Kim et al., 2009; Rajnicek et al., 1997; Ray et al., 2017; Teixeira et al., 2006).

36 Cell migration along surface structures such as ridges and grooves, is generally referred to as contact guidance and thought to play pivotal roles in neural development (Reig et al., 2014), tissue repair, immune response and cancer invasion (Friedl and Alexander, 2011). Nano- and micro-fabricated platforms have clarified how geometrical constraint affects focal adhesions distribution and actin stress fibers (Mathur et al., 2012; Oakley and Brunette, 1993; Ray et al., 2017). Focal complexes, substrate-anchoring clusters containing ECM-bound integrins that engage vinculin and talin associated with actin stress fibers, are restricted to be distributed within ridges and grooves (Franco et al., 2011; Ray et al., 2017). There alignment of stress fibers plays a major role in generating anisotropic contractility. While such a mechanism appears to be wide-spread in cells of epithelial or mesenchymal nature (Franco et al., 2011; Oakley and Brunette, 1993; Ray et al., 2017), topographical guidance in fast-moving amoeboid cells (Driscoll et al., 2014; Kwon et al., 2012; Sun et al., 2015; Wilkinson et al., 1982) which do not have stress-fiber and can migrate independently of cell-substrate adhesion (Lämmermann et al., 2008) is far less understood. Neutrophils are known to elongate along a few micron square grooves of a hemocytometer surface (Wilkinson et al., 1982). T-cells migrate along parallel ridges/grooves whose widths are hundreds of nanometers (Kwon et al., 2012). Adhesion-independent mode of migration in T-cells occurs under $2 \mathrm{D}$ confinement only if there is topographical asymmetry in the physical surrounding (Reversat et al., 2020). Macrophages are also known to spread along ridges and grooves (Wójciak-Stothard et al., 1996). Besides contractility, the other dominant determinant of directionality in fast-migrating amoeboid cells is the leading edge protrusion. While there are large body of work addressing how diffusible

56 chemoattractants determine when and where the leading edge forms to steer the cells, how they are guided by topography remains largely unknown (Sales et al., 2017).

The leading protrusion formed during cell migration has a large overlap in its molecular compositions with those formed during particle and liquid ingestion and thus the distinction between these processes are sometimes obscure (Heinrich and Lee, 2011). Conventionally, uptake of particle

61 and liquid are referred to as phagocytosis and macropinocytosis, respectively. As in the leading edge of migrating cells, macropinocytosis and phagocytosis involve large-scale conversion from 
64 complex for the side-branching nucleation (Molinie and Gautreau, 2018; Pollard, 2007). The 65 resulting actin polymerization generates the protruding force for the expanding edge of a cup-shaped 66 membrane invagination for ingestion (Jaumouillé et al., 2019; Rougerie et al., 2013). Phagocytosis 67 often refers to specific adhesion-dependent engulfment in immune cells, where the surface of the ingesting particle is decorated with opsonins; i.e. scaffold antigens or complements which through membrane-bound receptor signaling (Swanson, 2008) processively extends the protruding edge of the cup along the attached solid surface (Case and Waterman, 2015; Jaumouillé and Waterman, 2020). Macropinocytosis on the other hand refers to a self-organizing process where the shaping of the membrane by the branched actin meshworks does not require a solid surface (Swanson, 2008) and can occur constitutively (Williams and Kay, 2018). While this property makes it suitable for the uptake of nutrient media as well-known in cancer cells and Dictyostelium, macropinocytic particle uptake is also known for the entry of pathogenic bacteria into the host cells (Amara and Mercer, 2015). Non-opsonized polystyrene beads can also be ingested by amoeba Dictyostelium as well as macrophages and dendritic cells (Gilberti and Knecht, 2015; Mu et al., 2018; Pacheco et al., 2013). The receptor-independent cues that guide these macropinocytic/phagocytic membrane protrusion are still poorly understood.

Unlike endocytic cups mediated by clathrin, caveolin and BAR domain-containing proteins whose nanometer-scale topography dependence have been well studied (Galic et al., 2012; Zhao et al., 2017), phagocytic and macropinocytic cups involve global reorganization of actin cytoskeletons. In Dictyostelium, early organization of phagocytic/macropinocytic cup formation begins with the appearance of micrometer-size patches enriched in dendritic actin filaments whose inner-domain is characterized by strong accumulation of phosphatidylinositol (3,4,5)-trisphosphate (PIP3) (Hoeller et al., 2013) along with GTP-bound form of Ras and Rac. Depolymerizing factor coronin is distributed

87 further into the cytoplasmic side (Gerisch, 2010; Hacker et al., 1997; Maniak et al., 1995). These 88 active signaling patches are self-amplified by a positive feedback loop involving Ras and PI3K 89 (Fukushima et al., 2019; Sasaki et al., 2007; Taniguchi et al., 2013) and serve as a common precursor 90 or 'template' for phagocytic/macropinocytic cup formation (Gerisch et al., 2009; Veltman et al., 2016). 91 As the patch increase in size, its outer edge enriched in the SCAR/WAVE complex protrudes outward 92 to form a circular ruffle (Veltman et al., 2016). Loss of RasGAP NF1 or IqgC enhances both 93 phagocytosis and macropinocytosis (Bloomfield et al., 2015; Marinović et al., 2019; Williams and 94 Kay, 2018) indicating that Ras act positively on both processes. While deletion or pharmacological 95 inhibition of PI3K suppresses liquid uptake, deleterious effect on phagocytosis is limited to the uptake 
96 of large particles (Buczynski et al., 1997; Chen et al., 2012; Hoeller et al., 2013). Patches with

97 identical molecular organization are observed in the ventral side facing the substrate where they

98 appear as traveling waves (Asano et al., 2008; Bretschneider et al., 2009; Brzeska et al., 2016, 2014;

99 Taniguchi et al., 2013; Veltman et al., 2016). The ventral patches are thus thought to be a frustrated

100 form of a macropinocytic/phagocytic cup (Gerisch, 2010; Gerisch et al., 2009) similar to the frustrated

101 phagocytosis in macrophage placed on an opsonized surface (Barger et al., 2019; Masters et al., 2016).

102 In this work, to clarify the relationship between the surface microscale topography and actin patch

103 initiation, propagation and termination, live-cell imaging analysis of Dictyostelium on micro-

104 fabricated surfaces was performed. We demonstrate that the propagating ventral patches guide cell

105 migration along a micrometer-scale ridge in a PI3K-dependent manner. Quantitative analysis shows

106 that the nucleation of the patches occurs at the convex surface whereas its propagation is restricted at

107 the concave surface. Our results suggest that these properties allow the macropinocytic cup to

108 engulf extracellular fluid by default while at the same time directing it to faithfully trace bent and

109 bifurcating ridges when in contact with structured surfaces.

\section{RESULTS}

\section{Ventral actin patches propagate along microridges and orient polarized AX4 cells}

115 In order to first gain an over-view of the micro-topography dependency of Dictyostelium cell 116 deformation, we studied an aggregation-stage axenic strain (AX4) expressing GFP-Lifeact on an SU-

1178 structured substrate with straight ridges. Here, the ridges employed were $1 \mu \mathrm{m}$ in height, $3 \mu \mathrm{m}$ in 118 width and placed in parallel at intervals of $3 \mu \mathrm{m}$ (see Materials and Methods). Based on time-lapse 119 confocal imaging, cells were manually scored at each time frame for the presence of intense patches 120 of F-actin on the ventral plasma membrane. On both flat and structured surfaces, the percentage of 121 the cells that exhibited the patch increased from two hours after plating (Fig. S1A). The maximum 122 percentage of patch-positive cells were $79 \pm 13 \%$ and $98 \pm 1 \%$ for flat and micro-structured 123 surfaces, respectively (Fig. S1A, right panel, $250 \mathrm{~min}$ ). Cellular movement on a flat surface was 124 almost isotropic in direction (Fig. 1A, Fig. S1B). In the presence of ventral F-actin patches, cells 125 showed relatively small net displacement compared to those without (Fig. S1B). Actin patches 126 propagated along the ventral plasma membrane and the area in contact with the substrate expanded 
127 as the front traveled outward at the edge (Fig. 1B, Movie. S1). The direction of patch propagation

128 frequently changed as shown in Fig. 1B (red trajectories). On the other hand, cells on the micro-

129 structured surface migrated persistently along the ridges in the presence of patches (Fig. 1C, Fig.

130 S1C). As in patches found on flat surfaces (Asano et al., 2008; Bretschneider et al., 2009), F-actin

131 was most densely accumulated at the outermost edge of the patch which surrounds the inner territory

132 enriched in phosphatidylinositol(3,4,5)-trisphosphate $\left(\mathrm{PIP}_{3}\right)$ (Fig.1D, see also Supplementary Info Fig.

133 S3A-B). The cells were locked-in to a ridge and their movement seldom deviated from a single

134 linear track. The actin patches remained in the cell anterior as the cell moved forward along the

135 ridge (Fig. 1D, Movie. S2). More than $80 \%$ of migratory direction were oriented parallel to the

136 ridges in patch-positive cells on the micro-structured surface (Fig 1E). In contrast, the speed of cells

137 was not largely affected (Fig. 1F). On a flat surface, the persistence time of cell migration was 0.54

$138 \min (\mathrm{N}=23$ cells $)$ in the presence of ventral patches, compared to $3.0 \min (\mathrm{N}=36$ cells $)$ in the

139 absence of patches (Fig. 1G; Flat, patch(+), Flat, patch(-)). On the structured surface, persistence

140 time 13.7 min of migratory direction in the presence of ventral patches $(\mathrm{N}=34$ cells) was three times

141 higher compared to $4.6 \mathrm{~min}$ in the absence $(\mathrm{N}=21$ cells) (Fig. 1G; Struc., patch(+), Struc., patch(-)).

142 The F-actin patch and the leading edge traveled along a single ridge and rarely traversed to

143 neighboring ridges. While some cells migrated in one direction for over 30 minutes before

144 switching to the opposite direction, others frequently made turns and consequently showed small net

145 displacement; less than $80 \mu \mathrm{m}$ for $50 \mathrm{~min}$ (Fig. S1D). Change in the direction of cell migration was

146 accompanied by either patch reversal (Fig. S1E) or splitting (Fig. S1F). In patch reversal, the actin

147 patch starting from the cell anterior traveled to the opposite end (Fig. S1E). In patch splitting, the

148 anterior actin patch split in half and a daughter patch reached the posterior end and became a new

149 front while the other patch disappeared (Fig. S1F). Cells that exhibited frequent patch reversal and

150 splitting showed small net displacement (Fig. S1G). While these observations indicate strong

151 correlation between direction of cell movement and patch propagation, persistent migration was

152 rarely observed in growth-stage cells. There, almost the entire ventral side of the plasma membrane

153 was covered by a single continuous patch or a few separate patches, resulting in large ruffles projected

154 in many directions (Fig. S2A, Flat). Small patches were restricted at the ridges (Fig. S2A, Ridge,

155 24-48 sec) while larger ones often covered several ridges (Fig. S2A, Ridge, 72-120 sec). Regardless

156 of the presence of actin patches, persistent migration along the microridge rarely occurred (Fig. S2B,C,

157 compare to Fig. 1E) indicating that micro-topograhical features guide patch propagation but requires

158 additional cell polarity for persistent migration. 
These observations raise a question whether the F-actin/PIP3 patch formation which is thought to be a constitutive process that serves as a precursor for the macropinocytic/phagocytic cup formation (Gerisch et al., 2009; Veltman et al., 2016) was replaced by another distinct process when presented with curved surfaces. A previous work has shown that, in growth-stage or cells early into differentiation, the F-actin patches are extinguished by treating the cells with PI3kinase inhibitor LY294,002 (Taniguchi et al., 2013). We found that in aggregation-stage cells too, F-actin patches are extinguished with LY294,002 treatment in a dose-dependent manner (Fig. 2A). When cells migrating along the SU-8 ridge is applied locally with LY294,002 using a microneedle, F-actin patches disappeared immediately and the cell trajectories began to deviate from the ridge (Fig. 2BC). Directional bias decreased to a level comparable to non-treated cells without the ventral patch (Fig. 2D, Fig. 1E; Struc., patch(-)). A mock treatment neither extinguished patches nor impaired the topographic guidance (Fig. 2D). The results indicate that the micro-topographic guidance is PI3kinase-dependent and thus distinct from PI3kinase-independent, biased cell migration along much finer submicrometer-scale ridges (Sun et al., 2015). Spatial organization of the molecular components was also indistinguishable (Fig. S3) from those known for the ventral patches on flat surfaces (Schroth-Diez et al., 2009; Taniguchi et al., 2013; Veltman et al., 2016).

\section{PI3K signaling is induced by microtopography}

In order to see whether the micro-topography potentiates the appearance and lifetime of the patches, we first counted the positions of patch nucleation relative to the surface topography. The result shows that most ventral actin patches were initiated at the ridge in both growth- and aggregation-stage cells (Fig. 2E; "AX4, veg", $\mathrm{N}=28$ patches; "AX4, agg", $\mathrm{N}=27$ patches), independent of the position of the cell centroids (Fig. 2E, bottom). The duration of the patchpositive phase in growth-stage cells was on average $244 \pm 54 \sec (\mathrm{N}=20$ events) and $654 \pm 111$ $\sec (\mathrm{N}=24$ events $)$ on flat and ridged surfaces, respectively. In aggregation-stage cells, majority of patches persisted throughout our timelapse observations (50 min). Conversely, when the surface was coated with lectin wheat germ agglutinin (WGA), which promotes attachment of Dictyostelium cells to the substrates (Yoshida et al., 1984), the occurrence of actin patches decreased markedly (Fig. $\mathrm{S} 4 \mathrm{~A}, \mathrm{~B}$ ) and lifetime decreased to $4.0 \pm 0.6 \mathrm{~min}$ (structured, $\mathrm{N}=22$ patches; flat: no patch data), and the directional bias also diminished (Fig. S4C, compare to Fig. 1E). An earlier study has shown that the signaling patches that appear in the ventral membrane are the results of exaggerated Ras 
191 activity in the axenic cell-lines due to its null-mutation in RasGAP NF1 (Veltman et al., 2016).

192 Therefore we tested the patch properties in the parental non-axenic NC4 strain which has the intact

193 RasGAP. On a flat non-structured surface, only a few percent of NC4 cells exhibited ventral F-actin

194 patch $(4.3 \%, \mathrm{~N}=23$ cells), in agreement with the recent report (Veltman et al., 2016). In contrast,

195 we found that the percentage increased drastically on a microridged surface $(67 \%, \mathrm{~N}=18$ cells $)$.

196 The occurrence of patches were $0.35 \pm 0.08 / \mathrm{min}$ and $0.03 \pm 0.03 / \mathrm{min}$ for structured and non-

197 structured surfaces, respectively (Fig. 2F). Moreover, majority of the patches were initiated at the

198 ridge (Fig. 2E, "NC4", $\mathrm{N}=45$ patches), indicating that the surface ridge geometry serves as an

199 essential trigger for the patch initiation in NC4 cells. These patches were relatively short-lived

200 (Fig. 2G, Struc.: $21 \pm 2 \mathrm{sec}, \mathrm{N}=42$ patches, Flat: $34 \pm 5 \mathrm{sec}, \mathrm{N}=8$ patches) and did not facilitate

201 migration, however competed with the leading edge extension (Fig. 2H; $t=15 \sim 30$ sec, Fig. 2I; red

202 arrow). In aggregation-stage NC4 cells, F-actin patches were not detected under our conditions.

203 These results indicate that both the onset and the lifetime of F-actin patches are topography-dependent.

204 To further study the nature of topographical dependency, we employed NC4 expressing $\mathrm{PH}_{\mathrm{CRAC}}$

205 GFP as a marker for PIP3. Surprisingly, in the LatA-treated growth-stage NC4 cells, the patch in

206 the ventral plasma membrane was already present on the flat surface (Fig. 2J, right, Fig. 2K, right; N

$207=14$ cells) in addition to the structured surface (Fig. 2J, left). This stands in contrast to the absence

208 of patches on the flat surface in the untreated cells (Fig. 2F). On the structured surface, LatA-treated

209 cells were dislodged from the ridge and usually found between the two neighboring ridges, and the

210 PIP3 patches at the ventral-side were initiated above the ridge (Fig. 2J, left) which is consistent with

211 the ridge-dependence in non-treated cells (Fig. 2F). These patches remained at the site of initiation

212 and disappeared spontaneously (Movie. S3). The frequency map of the $\mathrm{PH}_{\mathrm{CRAC}}$-GFP patches along

213 the ventral-side of the plasma membrane shows a strong bias in their occurrence at the ridge (Fig. 2K,

214 left; $\mathrm{N}=15$ cells). Taken together with the presence of ventral PIP3 patches under LatA treatment

215 in AX4 (Taniguchi et al., 2013), the results indicate that PI3kinase and its upstream Ras are central

216 to the patch dynamics (Fukushima et al., 2019) and they are strongly dependent on micro-topography.

217 The high directedness of topographically guided cell movements raises a question about their

218 potential crosstalks with chemotaxis and the role of PI3K. Upon binding of cAMP to a G-protein

219 coupled receptor, transient activation of PI3K takes place over the course of a few minutes.

220 However, because null mutant of PI3Ks are still able to chemotax (Hoeller and Kay, 2007), the role

221 of PI3K is not entirely clear. When cells undergoing topography guidance were exposed to a

222 concentration gradient of cAMP formed from the tip of a glass needle, two distinct types of response 
223 were observed (Fig. 3A-D, Movie. S4). In approximately half of the case, F-actin patches

224 disappeared within 5 minutes after cAMP stimulation (Fig. 3E; $37.5 \%$ on the microstructured

225 surfaces $(\mathrm{N}=40$ cells $)$ and $58.5 \%$ on the flat $(\mathrm{N}=41$ cells $))$, and the cells migrated up the cAMP

226 gradient (Fig. 3A). For the rest of the case, actin patches persisted and cells continued to migrate

227 along the ridge irrespective of the orientation of the gradient (Fig. 3B). Trajectories of

228 representative 10 cells are shown in Fig. 3C (on flat surfaces) and 3D (on microridges), clearly

229 indicating the distinct chemotactic behaviors according to the presence of F-actin patches. The

230 percentage of cells with patches declined more rapidly in cells on the flat surface compared to those

231 on the structured surface (Fig. 3E). After $10 \mathrm{~min}$, patches are restored in cells on the structured

232 surface while they remain diminished in cells on flat surfaces which further supports the inductive

233 role of topography in the patch formation. The mean migration speed toward the cAMP source was

234 close to zero in patch-positive cells (Fig. 3F). The same cell was observed to switch between the

235 two behaviors; cells that first migrated up a cAMP gradient (Fig. S5A, 0-100 sec) stopped as a ventral

236 actin patch appeared (Fig. S5B, 110-210 sec), then resumed chemotaxis as soon as the patch

237 disappeared (Fig. S5B, 220-300 sec). In addition, small protrusions toward the cAMP source were

238 observed in some cells during micro-topographic guidance (Fig. S5C), suggesting that patch-positive

239 cells can still respond to extracellular cAMP, however some cells cannot override the topography

240 guidance. While the variable responses are not unexpected considering that there are cell-cell

241 heterogeneity in the expression level of the GPCR-signaling pathway, the erasure of the patch upon

242 cAMP stimulation in majority of the cells and its later recovery (Fig. 3E) suggest that the transient

243 activation of PI3K by cAMP can serve to release cells from directionality imposed by the topography

244 at least temporarily.

246 Substrate curvature determines the F-actin intensity and the propagation direction of the 247 signaling patches.

248 To further address geometrical features of the substrates that constrain the direction of patch 249 propagation, we employed an SU-8 surface with a large plateau and analyzed the patch dynamics 250 along its well-separated convex and concave corners. If the height of plateau $(h)$ was large enough 251 to prevent patches from covering both the top and bottom plane at the same time $(h=8.5 \mu \mathrm{m})$, we 252 found that patches propagated along the convex edge (Fig. 4A). For lower plateaus $(h=3.5 \mu \mathrm{m})$, 253 patches that traveled down from the top and reached the bottom (Fig. 4B, 00:00 01:00) did not 254 move across the concave edge. Rather than continuing to spread across the lateral plane, it always 
255 turned in the orthogonal direction and travelled along the edge (Fig. 4B, 01:00 01:30). These

256 observations suggest two opposing effects by the surface topography; convex surfaces attract and guide the patch, while concave surfaces prevent it from propagating further. For more rigorous quantification of this effect, reconstructed 3D confocal images were analyzed for spatial occupancies of patches with regard to the surface topography (Fig. 4C). Here, $D_{U}$ and $D_{H}$ are the distances from the convex edge to the farthest points covered by the actin patches at the top and lateral planes, respectively. $D_{L}$ is the distance from the concave edge to the farthest point within the patches at the bottom plane, and $D$ is the sum of $D_{U}, D_{H}$ and $D_{L}$. We found that, for $h=8.5 \mu \mathrm{m}, D_{U} / D=48 \pm 1 \%$ and $D_{H} / D=51 \pm 1 \%$ (Fig. $4 \mathrm{D}, \mathrm{N}=83$ plots), meaning that patches expanded equally well towards the top and lateral planes. At the intermediate height $h=3.5 \mu \mathrm{m}, D_{U} / D$ increased to $59.4 \pm 0.9 \%$ while $D_{H} / D$ decreased to $35.8 \pm 0.7 \%$ and $D_{L} / D=4.9 \pm 0.5 \%$ (Fig. 4D, $\mathrm{N}=117$ plots). Because $D_{U} / D>\left(D_{H}+D_{L}\right) / D$, the concave edge must be inhibitory. Note that, by definition, $D_{U} / D=\left(D_{\mathrm{H}}\right.$ $\left.+D_{\mathrm{L}}\right) / D$ at $h>0$ means no confinement effect. For a low plateau $h=1 \mu \mathrm{m}, D_{L} / D$ increased to 23.8 $\pm 0.6 \%$ (Fig. 4D, $N=407$ plots), reflecting the spread of the patches at the bottom plane.

The analysis above indicates that the convex edge traps the patch while the concave edge blocks it from propagating further. The near vertical contact angle between the dorsal side of membrane and the bottom plane (Fig. 1D, bottom; Fig. S3B) indicates high membrane tension. We postulated

272 that strengthening of crosslinked actomyosin meshwork at the dorsal plasma membrane may act to 273 suppress expansion of the F-actin patch filled with the branched actin meshwork. Since crosslinkers 274 of cortical actin - myosin II, cortexillin I and cortexillin II are the main source of cortical tension in 275 Dictyostelium (Kee et al., 2012; Reichl et al., 2008), the patches may not be prevented from traveling 276 across the concave edge in their null mutants. In support of this notion, $D_{L} / D$ for $h=1.5 \mu \mathrm{m}$ 277 increased from $9.9 \pm 0.4 \%$ in AX4 to $19 \pm 1 \%$ in ctxI-/ctxII- and $25 \pm 2 \%$ in $m h c A$ - (Fig. 4E). 278 Furthermore, we found that the ventral F-actin patches in $c t x I-/ c t x I I-$ and $m h c A$ - were less confined 279 to the microridge (ctxI-/ctxII- in Fig. 6A, 0-150, $750 \mathrm{sec}$; $m h c A$ - in Fig. S6A). Notably, in $m h c A-$ 280 patches often traversed the ridge and the bottom plane (Fig. S6B, C). These observations suggest 281 the cortical actomyosin at the dorsal side is essential for the confinement of F-actin patches to the 282 concave edge.

283 Since fabrication of ridges of various curvatures in z-direction requires fine 3-D 284 photolithography and thus technically demanding, we employed microridges of the same dimension 285 in z-direction however with patterned ridges so as to realize various curved corners in the x-y plane. 286 For square zig-zag patterns with alternating \pm 90 degrees corners (Fig. 5A, Movie. S5), we observed 
287 that the cell anterior and the underlying F-actin patch faithfully traced the zig-zag pattern. The

288 movement can be consistently understood from the topography dependence of the F-actin patch.

289 During turning, strong accumulation of F-actin continued along the outer corner, while it diminished

290 at the inner corner (Fig. 5B; $-90 \sim-30 \mathrm{sec}$ ) before it recovered as the leading edge exited the corner

291 (Fig. 5B; $0 \sim 90 \mathrm{sec}$ ). Fluorescence intensities of GFP-Lifeact increased transiently at the outer

292 corner by 1.6-fold (Fig. 5C; 90 degrees) and decreased down to 0.2-fold at the inner corner (Fig. 5D;

29390 degrees). Similar experiments were then performed using ridges with corners set at \pm 120 degrees

294 angle. There, the intensity fluctuations were smaller (Fig. 5C; 120 degrees), indicating that corners

295 with sharper angles are more effective in enhancing F-actin accumulation. Because the patch can

296 interface with more than one edge, we also tested how they respond when encountered with

297 inconsistent corners at each side. On a ridge with T-junctions, actin patches that entered the junction

298 from the bottom of the $\mathrm{T}$ faces two 90-degrees corners facing the opposite directions. There, the

299 patch stalled at both sides of the ridge and sometimes reversed its direction $(17.2 \%, \mathrm{~N}=29$ events,

300 Fig. S7A(a)). When the actin patches and the cell anterior entered the junction from the top of the

$301 \mathrm{~T}$, patches continued to propagate at the straight side while they were stalled at the concave side (Fig.

$3025 \mathrm{E})$. The percentage of patches that reversed its direction was $15.5 \%(\mathrm{~N}=84$ events, Fig. S7A(b),

303 "Reverse"). We also tested X-junctions, which also have concave corners at both sides (Fig. 5F,

304 Movie. S6). As in T-junctions, patches stalled as cells entered the intersection (Fig. 5F; $0 \sim 180 \mathrm{sec}$ ),

305 then began to propagate in the reverse direction (Fig. 5F; $180 \sim 360 \mathrm{sec})(27.6 \%, \mathrm{~N}=76$ events, Fig.

$306 \mathrm{~S} 7 \mathrm{~A}(\mathrm{c})$ ). In Y-junctions, reversal also occurred in $29.3 \%$ of the AX4 (Fig. S7B, "Reverse", $\mathrm{N}=58$

307 events). Patch reversal at the Y-junction was never observed for $c t x I$ - and ctxI-/ctxII- (Fig. S7B,

308 "Reverse", $\mathrm{N}=43$ and 57 events, respectively). These results further vindicate that concave surface

309 suppresses F-actin patches and that this inhibitory effect depends on the cortical actin at the dorsal 310 plasma membrane.

311 In addition to turning, traveling patches frequently split at the junctions (Fig. S7A, B, "Split").

312 For quantification, we employed the Y-junction (Fig. 6A, Movie. S7) since its three-fold symmetry

313 made data-sampling more efficient than the T-junction. Out of all cells that entered the Y-junction,

$31440.0 \%$ in AX4, $86.0 \%$ in $c t x I$ - and $75.4 \%$ in ctxI-/ctxII- ended up splitting (Fig. S7B, "Split"). As

315 bifurcated patches continued to propagate and extend the membrane along the respective branch (Fig.

$3166 \mathrm{~A}, 150 \sim 450 \mathrm{sec}$ ), expansion of the leading edges slowed down and came to a halt when one of the 317 competing patches disappeared (Fig. 6B, $340 \sim 600 \mathrm{sec}$ ). As soon as a patch diminished on one side, 318 the cell body rapidly retracted towards the surviving branch (Fig. 6A, 600 750 sec). Despite large 
319 stretching, there was no cell division or fragmentation as observed in giant cells fused by electric

320 pulses (Flemming et al., 2020). To gain an insight into the process of the ridge selection, the time

321 evolution of patch size and cell elongation along the respective branch were analyzed (Fig. S7C-E).

322 In all strains studied, AX4, ctxI- and ctxI-/ctxII-, split patches were asymmetric in size from the

323 beginning till the end, and the larger patch survived in the majority of cases (Fig. S7D). The

324 maximal distance from the junction point to the bifurcating cell edge (Fig. 6A, $t=600$ inset) in the

325 side of the surviving patch $l_{s, M A X}$ was always larger than those of the diminished patch $l_{d, M A X}$ (Fig. $6 \mathrm{C}$,

326 Fig. S7E). In $c t x I$ - and $c t x I-/ c t x I I-$, both $l_{s, M A X}$ and $l_{d, M A X}$ were long compared to AX4 (Fig. 6C).

327 The relative elongation $\left(l_{s, M A X}+l_{d, M A X}\right) / l_{0}$ was $1.29 \pm 0.08,1.59 \pm 0.09$ and $2.3 \pm 0.1$ for AX4, ctxI-

328 and $c t x I-/ c t x I I-\left(\mathrm{N}=23,32,36\right.$ events), respectively. $\quad l_{0}$ is the front-to-back length of a cell prior to

329 splitting. The patch was less confined to the ridge and thus spread at the bottom plane (Fig. 6A, 0

$330 \sim 150 \mathrm{sec}$ ). As the patch split, they were retracted to the side of the ridge (Fig. 6A, $300 \sim 450 \mathrm{sec}$ ).

331 As soon as the patch disappeared on one side, the surviving patch spread out to the bottom plane (Fig.

$3324 \mathrm{~A}, 750 \mathrm{sec})$. The observation suggests that the elevated tension exerted by the bifurcated

333 protrusions help confine the patch to the ridge even when cortical actomyosin is reduced. Indeed,

334 the cell area outside the ridge in the splitting patches normalized to that in the non-splitting patches

335 was $A_{S} / A_{N S}=0.68 \pm 0.04(\mathrm{~N}=14$ cells $)$ and $0.71 \pm 0.04(\mathrm{~N}=17$ cells $)$ in ctxI- and ctxI-/ctxII-,

336 respectively compared to $1.06 \pm 0.04(\mathrm{~N}=8$ cells) in AX4 (Fig. 6D, E). These measurements

337 suggest that the patch competes with tension-based repression which may not strictly require the

338 presence of cross-linked actin meshworks.

339 Overall, the above results indicate propensity of the patch to position itself above convexly

340 curved surfaces, and either be blocked or extinguished at the concavely curved surfaces. Besides

341 the patch dynamics, we asked whether the membrane protrusion itself can capture the ridge. In

342 immune-cells, phagocytosis involves a zippering mechanism where the advancing rim of a cup

343 protrudes sequentially by forming an anchorage with the specific surface signal, and the membrane

344 maintains close contact with the surface to gain traction (Jaumouillé and Waterman, 2020; Swanson

345 and Baer, 1995). Macropinocytic cup formation, on the other hand, does not require such specific

346 anchorage and traction (Jaumouillé and Waterman, 2020; Swanson and Baer, 1995). To see

347 whether non-zipper type cup formation is able to capture a ridge, we tested a minimal model of 348 macropinocytic cup formation (Saito and Sawai, 2020). The model describes a reaction-diffusion

349 process at the plasma membrane that forms propagating signaling patches that grow in size until they

350 consume a finite resource; e.g. total number of Ras molecules, actin nucleators, etc. The protruding 
351 force is perpendicular to the membrane, and it is restricted to the edge of a patch, which is plausible

352 in light of the SCAR/WAVE complex localization (Bretschneider et al., 2004) and the alignment of

353 actin filaments at this region (Jasnin et al., 2019). Although the model predicts that the geometry of

354 the patch patterning should naturally displace the position of the SCAR/WAVE complex from the

355 edge of the protrusion to yield the necessary inward tilt for cup formation (Saito and Sawai, 2020),

356 other processes that could yield the inward force should serve well for the present purpose. On flat

357 surfaces, the cups cannot form due to large load by the physical barrier (Fig. 7A-C). At the ridge,

358 protrusions are released from the frustrated state and successfully captured the ridge (Fig. 7D-F,

359 Movie. S8). Note that, in order to test the minimal requirement, adhesion strength between the

360 membrane and the surface was assumed to be uniform, and no topography dependence of the patch

361 dynamics was included. The simulations demonstrate that the primary motive force in a ring-like

362 profile is sufficient to trap the patch and hence lock the overall cell orientation.

\section{Discussion}

366 Previous works have suggested that signaling patches of PIP3 and Ras in Dictyostelium are 367 constitutive process that serve as templates for phagocytic/macropinocytic cups (Gerisch et al., 2009;

368 Veltman et al., 2016). The present work demonstrated that the patch dynamics are topography369 dependent and the resulting topography guidance serves to steer membrane protrusions along 370 micrometer-scale convoluted surfaces. The topographical guidance was PI3K-dependent and was 371 mediated by the following three properties: 1) F-actin independent patch initiation and growth that is 372 selective to convex surface of micro-meter scale, 2) patch confinement at the concave surface and 3) 373 physical capturing of the ridge by the membrane protrusion. The patch confinement was not 374 unexpected given the fact that the ventral F-actin waves are trapped at the sidewall of perforated 375 microwells (Jasnin et al., 2016) and that phagocytic cups are known to stall at the furrow of a budding 376 yeast where IBARa is localized (Clarke et al., 2010). Phosphatidylinositol 3,4,5-trisphosphate 3377 phosphatase PTEN is known to accumulate at the aspirated region, where the positive feedback from 378 actomyosin potentiates its force-induced translocation (Pramanik et al., 2009). Heightened activity 379 of PTEN may recruit more cortexillin to the plasma membrane and the resulting crosslinked actin 380 meshwork should prevent expansion of the F-actin patch. The resulting membrane tension should 381 form a positive feedback loop to further suppress patch propagation at the concave edge. Such notion is in line with our observation that confinement at the concave surface was reduced in the null 
383 mutants of myosin II and of cortexillins (Fig. S6, Fig.6A). The fact that the reduction was rescued

384 when the cells were stretched at the bifurcating ridges (Fig. 6D-E) further strengthens the notion that

385 the patch confinement is mediated by membrane tension rather than directly through cortical

386 actomyosin. These features may be related to extinction of actin waves in neutrophils when they

387 collide with physical obstacles (Weiner et al., 2007) and the tension-mediated suppression of the leading edge in migrating cells.

On the other hand, the topography-dependent patch nucleation and the ridge capturing suggest a novel mechanism whereby a cup is selectively generated at the plasma membrane in contact with

391 sufficiently curved surfaces and the ability of the resulting ring-like protrusion to capture the ridge.

392 Since a misplaced patch would have high chance of being confined to the concave surface, it is 393 essential that a patch is selectively induced at the convex surface for efficient surface capturing.

394 Topography guidance was completely eliminated by LY treatment (Fig. 2B-D) indicating that the 395 patch nucleation at the ridge or the physical capturing itself or both are PI3K dependent. Since the

396 latter requires sustained presence of PIP3 patches, contributions from the two processes is difficult to 397 separate. Dictyostelium discoideum has five class-I PI3Ks, in which PI3K1/2 are essential to 398 nucleate PIP3 patches in the early stage of macropinocytic cup formation (Hoeller et al., 2013). 399 Activity of PI3Ks requires interaction with RasG, RasS and Rap at its Ras-binding domain (Hoeller 400 et al., 2013; Kortholt et al., 2010). Patch of pan-RAS-GTP probe Raf1RBD has been observed in 401 PI3K-null (Veltman et al., 2016) under LatA treatment (Fukushima et al., 2019) indicating that Ras is 402 the central driver of the patch dynamics. Since forced elevation of Ras/Rap activity can elevate 403 spontaneous patch generation (Miao et al., 2017), the present analysis indicates that surface 404 topography has a similar effect on Ras/PI3K but acting locally. In the present study, topographical 405 dependence was strictly observed in the wild-type NC4 strain (Fig. 2E-F) which is poor at liquid 406 uptake. Even in the derivative axenic strain which has hyper Ras activity and can constitutively 407 generate patches on flat surfaces (Bloomfield et al., 2015; Veltman et al., 2016), our results 408 demonstrated that, when presented with microridges, patch initiation occurred exclusively at the 409 ridges (Fig. 2E) suggesting that, topographical-sensing can operate on top of the elevated Ras/PI3K 410 activity. Deletion of all five PI3Ks in Dictyostelium significantly decreases the ingestion rate for 411 yeast particles of 3-5 $\mu \mathrm{m}$ in diameter (Chen et al., 2012) but not for bacteria or $1 \mu \mathrm{m}$ latex beads 412 (Hoeller et al., 2013). Interestingly, micrometer-scale dependencies are also seen in other systems. 413 Phagocytic uptake of IgG-coated beads in RAW264.7 cells requires PI3K when beads are larger than $4143 \mu \mathrm{m}$ in diameter (Cox et al., 1999). In the frog egg extracts, the rate of actin polymerization on a 
$415 \mathrm{PI}(4,5) \mathrm{P} 2$ coated glass beads is $2-3$ times higher for $1 \mu \mathrm{m}$ radius beads compared to $150-400 \mathrm{~nm}$

416 radius beads (Gallop et al., 2013). Taken together with our present findings, the coordinated

417 engagement of Ras and PI3K in amplifying cytoskeletal signaling in a topography-dependent manner

418 maybe a wide-spread mechanism for large particle uptake.

419 It should be noted that the PIP3/F-actin patches described here takes place in the order of minutes

420 and a few micrometers in size. These features contrast with the much faster membrane curvature-

421 dependent waves in mast cells which takes place in the order of seconds in association with F-BAR

422 dependent nanoscale endocytosis that are synchronized in time and space (Wu et al., 2018; Yang et

423 al., 2017). Strengthening the contact between the substrate and the plasma membrane almost

424 completely abolished the F-actin patches both on the flat and structured surfaces (Fig. S4B). If the

425 patch induction is based on spontaneous curvature of lipid and membrane bound molecules (Gov,

426 2018), such suppression is expected as strong adhesion will flatten the membrane. Although this is

427 consistent with our observation on adhesive flat surface, the same was true for adhesive microridged

428 surface suggesting that strongly adhesive conditions may directly be inhibitory to patch initiation,

429 propagation or both. Despite similarities in the clutching machineries with the mammalian

430 counterparts, Dictyostelium lacks real integrin and is able to gain traction without specific

431 extracellular ligands for the adhesion complex. Cell-substrate adhesion in Dictyostelium is mediated

432 by non-specific van der Waals force (Kamprad et al., 2018; Loomis et al., 2012) that is assisted by

433 integrin-beta like adhesion protein SibA that forms a complex with a kinase Phg2 (Froquet et al.,

434 2012), another adhesion molecule SadA, paxillin, vinculin, two talin homologues TalinA or TalinB

435 (Tsujioka et al., 2012). Rap is required for PI3K activation as well as Ras-binding domain

436 containing Phg2 kinase (Gebbie et al., 2004; Kortholt et al., 2006). Both Phg2 and SadA are known

437 to be essential for phagocytosis (Fey et al., 2002; Gebbie et al., 2004). In order to mediate adhesion,

438 SadA requires its cytoplasmic tail region that interacts with actin cross-linker Cortexillin I (Kowal

439 and Chisholm, 2011) which is absent from the patch (Fig. S4B)(Schroth-Diez et al., 2009). One

440 possibility is that cell-substrate adhesion at the patch is weak and that fluctuating membrane

441 undulation is required for the feedback amplification of the SCAR/WAVE complex (Huang et al.,

442 2013). In several mammalian cell lines, ventral F-actin waves require a cycle of integrin

443 engagement and disengagement to ECM (Case and Waterman, 2011). Inhibition of integrin

444 disengagement by addition of $\mathrm{Mn}^{2+}$ prevents wave propagation (Case and Waterman, 2011),

445 suggesting that cell-substrate adhesion needs to be somewhat loose and within a proper range for 
446 wave generation. A weak cell-substrate adhesion is also reported at the frustrated phagocytic cup

447 in macrophage (Barger et al., 2019).

$448 \quad$ F-actin waves appear in the leading edge of migrating neutrophils (Weiner et al., 2007) as well

449 as in neuronal extensions during the neurite outgrowth (Katsuno et al., 2015), however their role in

450 Dictyostelium migration has long been debated. Our work demonstrated a clear example of F-actin

451 wave directed migration in the axenic strain of Dictyostelium. This new mode of directed cell

452 migration which we shall refer to as 'phagotaxis' likely resulted from combination of ability of the

453 aggregation-stage Dictyostelium to polarize while still retaining ability to form phagocytic cup.

454 From lack of their presence in the aggregation-stage NC4 cells, migratory roles of ventral patches in

455 Dictyostelium is not clear. This puzzle parallels that for large-scale macropinocytosis in

456 Dictyostelium as extracellular environment that supports it is so far unknown (Kay et al., 2019). We

457 envisage that the natural habitat that supports efficient macropinocytosis; i.e. large patch formation

458 in non-axenic wild type is also likely to support phagotaxis. We note that vertically confined

459 Dictyostelium formed phagocytic cup sideways facing a yeast particle in contact (Fig. S8A). The

460 cup which was more persistent in the aggregation-stage cells (Fig. S8B) supported directed migration

461 as the cell pushed the particle forward. Such phagotactic movements may help cells transfer target

462 particles to a better location that supports ingestion. Similar mechanisms may underlie streaming

463 migration of macrophages in contact with target cancer cells (Sharma et al., 2012). These contact-

464 dependent migration are also reminiscent of contact activation of locomotion that supports streaming

465 cell aggregation and cell-type dependent cell sorting (Fujimori et al., 2019) during the multicellular

466 stage of Dictyostelium lifecycle. There, cell-cell contact signal is mediated by Ig-domain containing

467 transmembrane molecule TgrB1 and $\mathrm{C} 1$ acting in trans between the front and back of the neighboring

468 cells. Although TgrB1 and $\mathrm{C} 1$ are polymorphic genes that mediate kin-recognition in Dictyostelium

469 discoideum, no phagocytic behavior has been observed between non-compatible Tgr allotype. Lack

470 of clear Ras/PI3K activity at the contact site (Fujimori et al., 2019) suggests that TgrB1/C1 interaction

471 is disengaged from topography dependent behavior. Given the presence of other cell-cell adhesion

472 proteins and ECM in the later stage of development, phagotaxis may also have a role in 473 morphogenesis.

476 Materials and Methods

477 Plasmids, cell strains. 
478 Dicty-codon optimized mNeon (Tunnacliffe et al., 2018) and Lifeact was inserted into G418- and 479 Hygromycin-resistant plasmids pDM304 and pDM358 (Veltman et al., 2009) to obtain pDM304480 Lifeact-neon and pDM358-Lifeact-neon. Plasmid pDGFP-MCS-Neo-CI (Faix et al., 2001) was a 481 kind gift from Prof. Igor Weber. Plasmid pBIG-GFP-myo (Moores et al., 1996) was obtained from 482 Dicty Stock Center. Laboratory wild-type strain AX4 and mutant strains ctxI- (NBRP, S00100), ctxI-/ctxII- (NBRP, S00404) and $m h c A$ - (Dicty Stock Center, DBS0236379) were transformed following the standard electroporation protocol (Nellen et al., 1984). To generate PHcrac-GFP/NC4, NC4 cells washed free of bacteria were incubated overnight in HL5 medium before and after electroporation. Cells were then resuspended for selection in bacterial suspension in DB including $10 \mu \mathrm{g} / \mathrm{ml} \mathrm{G} 418$. The following strains were constructed: GFP-mhcA/PH $\mathrm{CRAC}_{\mathrm{C}} \mathrm{RFP} / \mathrm{Ax} 4$, Lifeactneon/PI3K1 ${ }^{\text {N1-487}}$-RFPmars/Ax4, Lifeact-neon/ctxI-, Lifeact-mRFPmars/GFP-CtxI/ctxI-, Lifeactneon/ctxI-/ctxII-, Lifeact-neon/mhcA- and Lifeact-GFP/NC4. Following strains are described previously (Fujimori et al., 2019; Taniguchi et al., 2013): GFP-Lifeact/Ax4, Lifeact-mRFPmars/Ax4,

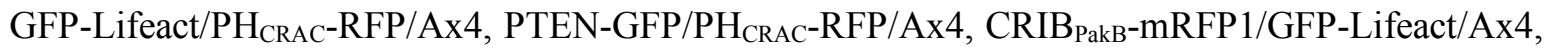

\section{Surface fabrication.}

497 Microstructured SU-8 surfaces were prepared by standard photolithography. The microridged 498 surface consisted of two layers of fabricated SU-8. A glass coverslip was washed with $\mathrm{NaOH}$ and 499 treated with air plasma. The first layer of SU-8 (MicroChem) was spin-coated on the coverslip and 500 baked at $65{ }^{\circ} \mathrm{C}$ for $1 \mathrm{~min}$, then at $95{ }^{\circ} \mathrm{C}$ for $3 \mathrm{~min}$. Photoresist SU-8 2 or SU-8 3005 were spin501 coated at $2000 \mathrm{rpm}$ to achieve thickness around $2 \mu \mathrm{m}$ and $5 \mu \mathrm{m}$, respectively. The substrate was 502 uniformly exposed with UV using an aligner (MA-20, MIKASA), baked at $65{ }^{\circ} \mathrm{C}$ for $1 \mathrm{~min}, 95{ }^{\circ} \mathrm{C}$ for $5033 \mathrm{~min}$ followed by $200{ }^{\circ} \mathrm{C}$ for $5 \mathrm{~min}$. After cooling down to room temperature, the second layer of 504 SU-8 was spin-coated at the same rotation speed as the first layer and baked at $65{ }^{\circ} \mathrm{C}$ for $1 \mathrm{~min}$, then 505 at $95{ }^{\circ} \mathrm{C}$ for $3 \mathrm{~min}$. The first and second layer were fabricated using the same photoresist. Chrome 506 masks (CBL4006Du-AZP, Clean Surface Technology) were patterned by a laser drawing device 507 (DDB-201-TW, NEOARK). The substrate was UV-treated over a chrome mask and baked at $65{ }^{\circ} \mathrm{C}$ 508 for $1 \mathrm{~min}, 95^{\circ} \mathrm{C}$ for $1 \mathrm{~min}$, etched using SU-8 developer and lastly hard-baked at $200{ }^{\circ} \mathrm{C}$ for $5 \mathrm{~min}$. 
509 The fabricated SU-8 substrate was for one-time use.

$510 \quad$ For construction of microgrooved glass substrates, glass coverslips (MATSUNAMI, No.1, 0.13

$511 \sim 0.17 \mathrm{~mm}$ in thickness) were uniformly coated with $\sim 100 \mathrm{~nm}$ thick chrome using a sputtering device

512 (E-200S, ANELVA), then the SU-8 with the microridges were attached on top which serves as a mask

$513(1.5 \mu \mathrm{m}$ in height and $3 \mu \mathrm{m}$ in width at an interval of $3 \mu \mathrm{m})$. The substrates were immersed into

514 chrome etchant to etch SU-8-uncovered chrome coatings. The glass substrates covered by chrome 515 and SU-8 ridges were etched by gas plasma $\left(\mathrm{Ar}: \mathrm{O}_{2}: \mathrm{C}_{4} \mathrm{~F}_{8}: \mathrm{CHF}_{3}=27: 1: 1: 1\right)$ using a dry-etching

516 system (NLD-5700Si, ULVAC). Residual contaminants were removed by ethanol, piranha solution

517 and chrome etchant. Before use, the microgrooved glass substrates were washed by ethanol and

$518 \mathrm{NaOH}$.

519 Microfabricated SU-8 and glass surfaces were measured using AFM (Nanowizard3, JPK, now 520 Bruker) or DektakXT (Bruker). AFM was installed in an inverted microscope (IX70, Olympus) on 521 active vibration isolator (Herz). Cantilevers mounted on AFM were Tap300-G (BudgetSensors) and 522 ACTA (AppNano). HS-500MG (BudgetSensors) with $500 \mathrm{~nm}$ step height was used as a height 523 calibration standard for AFM measurements. Prepared SU-8 and glass substrates were set at the 524 bottom of $\varphi 35 \mathrm{~mm}$ culture dish (MatTek) using a 9 × $9 \mathrm{~mm}^{2}$ frame seal (SLF0201, Biorad). The 525 chamber was plasma-treated to improve wettability, using a plasma cleaner (PDC-32G, Harrick 526 Plasma) immediately before plating cells.

\section{Cell preparation and timelapse imaging.}

529 Axenic strains of Dictyostelium discoideum were grown while shaken at $22^{\circ} \mathrm{C}$ in $\mathrm{HL} 5$ with $60 \mu \mathrm{g} / \mathrm{mL}$

530 hygromycin B, $10 \mu \mathrm{g} / \mathrm{mL}$ G418 where appropriate. Lifeact-GFP/NC4 and PHcrac-GFP/NC4 cells 531 were cultured in developmental buffer (DB) including E. coli $\mathrm{B} / \mathrm{r}$ at $\mathrm{OD}_{600}=6$. For live-cell imaging, 532 growing Dictyostelium cells were washed twice, resuspended in DB at $5 \times 10^{6}$ cells $/ \mathrm{ml}$ and shaken at $53322{ }^{\circ} \mathrm{C}, 155 \mathrm{rpm}$ for 1 hour. Cells were then pulsed with cAMP (final concentration $50 \mathrm{nM}$ ) every 6 $534 \min$ for 4.5 hours. Starved cells were plated at $\sim 3 \times 10^{3}$ cells $/ \mathrm{cm}^{2}$ on a fabricated SU-8 or glass 535 surface described above. For observation on glass surfaces (Fig. S3), adenylyl cyclase inhibitor 536 SQ22536 (Wako) was added at a concentration of $150 \mu \mathrm{M}$ to circumvent cell-cell agglutination 537 facilitated by the low adhesiveness of the glass surface. NC4 cells were collected by suspending in $538 \mathrm{DB}$, pelleted by centrifuged at $100 \mathrm{rcf}$ for $3 \mathrm{~min}$ and resuspended in DB. The step was repeated 539 three times to remove bacteria. Washed cells were plated at a density of $\sim 1 \times 10^{5}$ cells $/ \mathrm{cm}^{2}$. For 
540 observation with yeast, AX2 cells expressing Lifeact-neon and yeast Rhodotorula mucilaginosa

541 (NBRP, S90641) were loaded into polydimethylsiloxane (PDMS) chamber. The chamber was

542 fabricated as previously described (Nakajima et al., 2016). Images were obtained using an inverted

543 microscope (IX83 or IX81, Olympus) equipped with a laser confocal scanning unit (CSU-W1 or

544 CSU-X1, YOKOGAWA) and a EMCCD camera. For cAMP and LY-loading, $20 \mu 1$ of $100 \mathrm{nM}$

545 cAMP and $100 \mu \mathrm{g} / \mathrm{ml}$ Alexa594, or $1 \mathrm{mM} \mathrm{LY294,002} \mathrm{and} 10 \mu \mathrm{g} / \mathrm{ml}$ Alexa594 were prepared in DB

546 and loaded into Femtotips II (Eppendorf). The tip mounted on the micromanipulator (TransferMan

$5474 \mathrm{r}$ or TransferMan NK2, Eppendorf) was pressurized at 80-100 hPa using a microinjector (IM300,

548 NARISHIGE or FemtoJet, Eppendorf). All live-cell imaging was performed at $22^{\circ} \mathrm{C}$.

\section{Data analysis.}

552 Image analysis was performed using ImageJ, Python and Microsoft Excel. To quantitate the relationship between cell migration and the ventral actin patches (Fig. 1E-G), confocal and transmitted-light images of GFP-Lifeact expressing cells that were acquired using 20x objective lens at 1 min intervals for 50 min time-windows $(\mathrm{N}=6)$ were analyzed. To calculate the ratio of patchpositive cells, cells were manually assigned 1 or 0 according to presence or absence of the actin patches at each timepoint and averaged over all timepoints and cells (Fig. S1A). Cell trajectories were obtained by auto- or manual-tracking of cell centroids. The average speed and distribution of migratory direction relative to the ridge were calculated from the centroid displacement in moving time-window of 1 min (Fig. 1E, F). The relationship between the mean square displacement and time was fitted by the two parameters in the persistent random walk model (Dunn, 1983) to obtain 562 the persistence time (Fig. 1G).

For quantification of spatial distribution of ventral PIP3 patches in Latrunculin A-treated cells

564 (Fig. 2K), 3-D confocal images of PHCRAC-GFP expressing cells were acquired every $10 \mathrm{sec}$ for $>5$

565 minutes from $z=0$ (the basal surface) to $z=3 \mu \mathrm{m}$ at $z$-interval of $0.5 \mu \mathrm{m}$. From the maximum

566 intensity projection of Z-stacks, a region occupied by $\mathrm{PH}_{\mathrm{CRAC}-\mathrm{GFP}}$ patches and the cell outlines were

567 extracted and aligned against the average cell centroid positions. The aligned binary image stacks

568 of $\mathrm{PH}_{\mathrm{CRAC}}-\mathrm{GFP}$ patches and cell outlines were cropped to 180 x 90 pixels and averaged over 892

569 frames from 15 cells (Struc.) and 826 frames from 14 cells (Flat). The average intensity of PHCRAC

570 patch was normalized so that the total value of all pixels is 1 . Averaged cell outlines were calculated

571 in the polar coordinate system. 
To quantitate the height dependence of patch dynamics on the plateau (Fig. 4D), Z-stacked timelapse confocal images of cells expressing GFP-Lifeact were acquired every $10 \mathrm{sec}$. Images were projected into the $\mathrm{x}$-axis in Fig. $4 \mathrm{C}$ as maximum fluorescent intensity were displayed. In the projected images, $D_{U}, D_{H}$, lengths from convex corner to the patch edges within top and lateral surfaces, $D_{L}$, a length from concave corner to the patch edge within bottom surface and $D$, the sum of these three lengths, were measured at each timepoint. Ratio $D_{U} / D, D_{H} / D$ and $D_{L} / D$ were averaged over all timepoints from all cells and plotted. For quantification of the relationship between the angle of the ridge corners and F-actin accumulation (Fig. 5C, D), Z-stacked time-lapse confocal images of GFP-Lifeact expressing cells plated on the zig-zag ridges were acquired every 6 or 12 sec. From kymographs of GFP-Lifeact along the inner and outer corners, fluorescent intensities within actin patch regions were extracted and integrated at each timepoint. Time frame was aligned so that patch centroids reached the corner (Fig. 5B) at time 0 . The integrated fluorescence intensities were normalized to the value at $t=-120 \mathrm{sec}$ and averaged over all events. The membrane extension accompanied by two split patches and the patch diameters on the Y-shaped ridges were quantitated using kymographs along both branches (Fig. 6C, S7D, E). Lengths from junction-point to the two leading edges were defined as $l_{s}$ and $l_{d}$ (s: survived and $d$ : disappeared). Cell area outside the ridge (Fig. 6D, E) was measured using binary cell-mask images created from GFP-Lifeact or Lifeact-neon fluorescence images from which the region of ridges were subtracted. The remaining area was timeaveraged according to whether cells exhibited single patch $\left(A_{N S}\right)$ or two split patches $\left(A_{S}\right)$.

\section{Phase-field model}

An abstract field variable $\phi(\boldsymbol{r})$ describes the cell interior region $(\phi=1)$ and the exterior region $(\phi=0)$ in a 3-D coordinate $\boldsymbol{r} . \quad \phi$ is continuous and varies sharply at the interface with finite width characterized by the small parameter $\epsilon$. To describe the interfacial dynamics, we employed the following phase-field equation

$$
\tau \frac{\partial \phi}{\partial t}=\eta\left(\nabla^{2} \phi-\frac{G^{\prime}(\phi)}{\epsilon^{2}}\right)-M_{V}\left(V-V_{0}\right)|\nabla \phi|+F_{\text {poly }}|\nabla \phi|-A_{\text {rep }} \chi^{2} \phi+A_{\text {att }}|\nabla \chi||\nabla \phi|
$$

where $G^{\prime}=16 \phi(1-\phi)(1-2 \phi)$ and $V=\int \phi \mathrm{d} \boldsymbol{r}$. The first term in the right hand side represents curvature-driven force associated with surface tension $\eta$. The second term describes the 
603 effective elasticity where $V_{0}$ is the cell volume at the resting state and $M_{V}$ is a fixed positive

604 parameter. The third term describes the force normal to the interface driven by actin polymerization.

605 The magnitude of force $F_{\text {poly }}$ is a function of the local concentrations of signaling molecules as

606 described below. The interactions between the cell and the substrate are described in the fourth and

607 fifth term. The fourth term is the volume exclusion, and the fifth term describes the effective

608 adhesion. The microridged substrate is described by another field variable $\chi(\boldsymbol{r})$ as follows:

609

$$
\chi(\boldsymbol{r})=\frac{1+\tanh \left(\frac{z_{0}-z}{\varepsilon / 2}\right)}{2}+\frac{1+\tanh \left(\frac{z-z_{0}}{\varepsilon / 2}\right)}{2} \frac{1+\tanh \left(\frac{z_{0}+h-z}{\varepsilon / 2}\right)}{2} \frac{1+\tanh \left(\frac{L / 2+w / 2-x}{\varepsilon / 2}\right)}{2} \frac{1+\tanh \left(\frac{x-L / 2+w / 2}{\varepsilon / 2}\right)}{2} .
$$

612 Parameters are: the offset of the substrate $\left(z_{0}\right)$, width $(w=3.0 \mu \mathrm{m})$ and height $(h=1.5 \mu \mathrm{m})$ of the ridge 613 and the length in the x-direction of simulated space $L=40 \mu \mathrm{m}$. The length of the simulated space 614 in the y-direction was $60 \mu \mathrm{m}$.

615 For time development of the signaling molecule, we adopted the following reaction-diffusion equations of the activator molecule $(A)$ with limited total resource of molecule $\left(A_{\mathrm{t}}\right)$ :

622 where $D_{A}$ and $D_{I}$ are diffusion constants of $A$ and $I$ molecules, respectively. In the first 623 equation, $B$ represents inactive form of the activator molecule, which is assumed to diffuse 624 sufficiently fast and thus can be written as $B=A_{\mathrm{t}} / S-\langle A\rangle$, where $S$ is the cell surface area $S=$ $625 \int \psi / \varepsilon \mathrm{dr}^{3}$ and $\langle A\rangle$ is the total of $A$ divided by $S$.

627 To describe the plasma membrane region, we introduced an auxiliary phase-field $\psi=$ $628\left(1+e^{-\beta(\phi(1-\phi)-\theta)}\right)^{-1}$ which defines the interface between cell exterior $(\phi=0)$ and interior $(\phi=$ 629 1) region. By definition, $\psi=1$ represents the cell membrane and $\psi=0$ elsewhere. A 630 sufficiently large value of $\beta$ was chosen so that the interface is sharp. Small offset $\theta$ is given to 631 render $\psi$ non-zero at the interface. Using $\psi$, we arrive at the following equations: 


$$
\frac{\partial}{\partial t} \psi A=-\nabla \cdot(\psi A v)+\psi\left[\frac{A^{2} B}{1+I}-A\right]+D_{A} \nabla(\psi \nabla A)
$$

634

(4)

$$
\frac{\partial}{\partial t} \psi I=-\nabla \cdot(\psi I v)+\psi\left[k_{1} A^{2}-k_{2} I\right]+D_{I} \nabla(\psi \nabla I)
$$

636

$$
\boldsymbol{v}=-\left[\frac{\eta\left(\nabla^{2} \phi-\frac{G^{\prime}(\phi)}{\epsilon^{2}}\right)}{|\nabla \phi|}-M_{V}\left(V-V_{0}\right)+F_{\text {poly }}\right] \frac{\nabla \phi}{|\nabla \phi|} .
$$

642 Around the substrate, Eq. (4) is given additional noise term at rate $\lambda$ per volume. The spatial

643 profile of the noise is given by $\mathcal{N}(\boldsymbol{x})=\mathcal{N}_{0} \times \exp \left(-\frac{\left|\boldsymbol{x}-\boldsymbol{x}_{\mathrm{c}}\right|^{2}}{2 d^{2}}\right)$, where $d$ is the initial nucleation size,

644 and $\mathcal{N}_{0}$ is the noise intensity that follows an exponential distribution with the average $\sigma$.

646 We assume that the magnitude of protrusion force in Eq. (1) and (6) is facilitated by $A$ but attenuated 647 by $I$ following

$$
F_{\text {poly }}(A(\boldsymbol{r}))=F \frac{\left(A / K_{1}\right)^{n_{h}}}{1+\left(A / K_{1}\right)^{n_{h}}} \frac{1}{1+\left(I / K_{2}\right)^{n_{h}}}
$$
and Sawai, 2020).

654 Author contributions. GH and SS conceived the work, planned and managed all aspects of the project. GH performed all microfabrication, cell preparation, microscopy observations, and image cell lines. HY, TO, ST and AN assisted with microfabrication. NS formulated the mathematical 
660 Acknowledgements. The authors thank present and past members of the Sawai lab for various

661 technical and scientific inputs. We thank Prof. Igor Weber for pDGFP-MCS-Neo-CI, Prof. Jonathan

662 Chubb for Dicty-codon optimized NeonGreen, Prof. James Spudich and Dicty Stock Center for pBIG-

663 GFP-myo (DSC ID: 381), Prof. Hidekazu Kuwayama and the National BioResource Project (NBRP)

664 Nenkin for ctxI- (NBRP, S00100), Prof. Günther Gerisch, Dicty Stock Center and NBRP for ctxI-

665 /ctxII- (NBRP, S00404) and Prof. Douglas Robinson and Dicty Stock Center for $m h c A$ -

666 (DBS0236379). Takehiko Oonuki for the GFP-mhcA/PH ${ }_{\text {CRAC }}$ RFP/Ax4 cell line, Toyoko Sugita

667 and Nao Shimada for pDM304-MCS-neon and pDM358-MCS-neon constructs. This work was

668 supported by grants from Japan Science and Technology Agency (JST) CREST JPMJCR1923, Japan

669 Society for Promotion of Science (JSPS), Ministry of Education, Culture, Sports, Science and

670 Technology (MEXT) KAKENHI JP19H05801 to SS, Platform for Dynamic Approaches to Living

671 System from MEXT and Japan Agency for Medical Research and Development (AMED) and in part

672 by Joint Research by Exploratory Research Center on Life and Living Systems (ExCELLS) Grant

673 18-204, MEXT KAKENHI JP19H05416, JP18H04759 and JP16H01442; JSPS KAKENHI

674 JP17H01812 and JP15KT0076 (to S.S.). GH was supported by JSPS Fellowship Grant JP18J14678. 


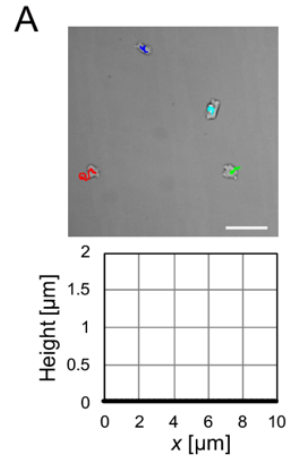

E

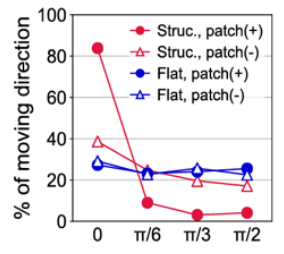

B

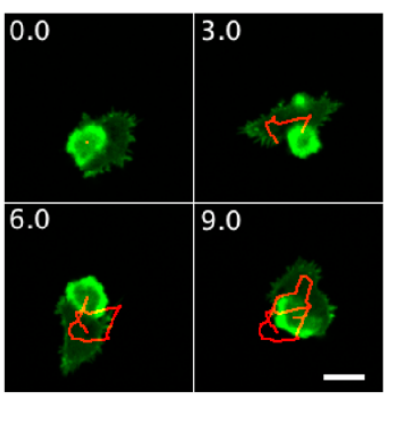

$\mathrm{F}$

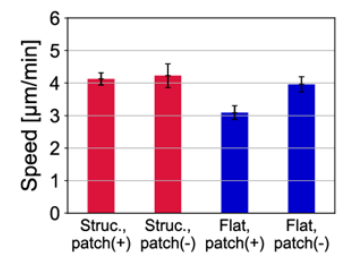

C

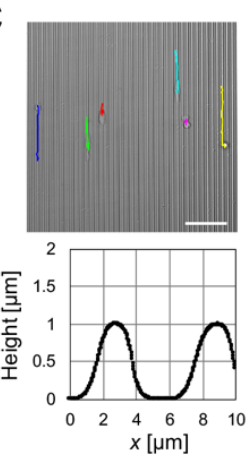

G

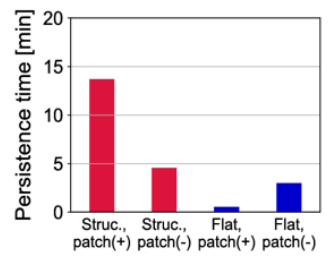

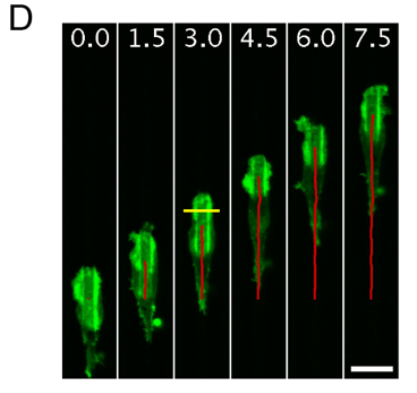

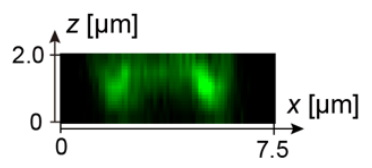

Figure 1. Guidance of ventral actin patches and membrane evagination in Dictyostelium AX4 cells on a microridged surface.

(A-D) Cell trajectories and the ventral F-actin patch dynamics in aggregation-stage AX4 cells on non-

B) and microstructured SU-8 surfaces

$(\mathbf{C}$ and $\mathbf{D})$

D).

(A and

C, upper panels)

Transmitted light images of a representative field of view. Colored lines: trajectories of individual

684 cells for $20 \mathrm{~min}$. Scale bars, $50 \mu \mathrm{m}$. (A and C, lower panels) Representative surface geometry.

685 (B and D) Time-lapse confocal images of the F-actin patch. Green: GFP-Lifeact fluorescence; $z$ -

686 slice near the SU-8 surface $(z=0)(\mathbf{B})$ and maximum intensity projection (MIP) from $z=0$ to $2 \mu \mathrm{m}$ (D, upper panel) and the cross-section along the yellow line (D, lower panel). Red lines: centroid

(E) Angular distribution

691 Persistence time of cell displacement. 
A

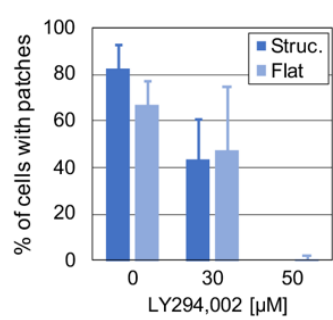

E

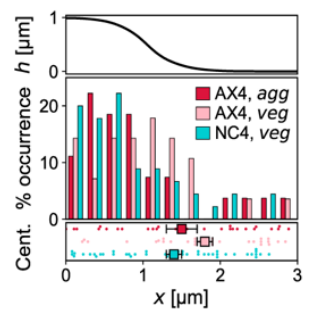

$\mathrm{H}$

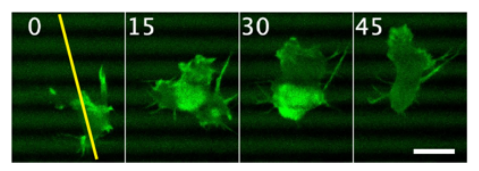

B

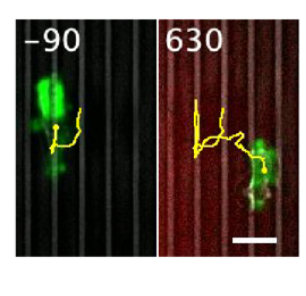

C

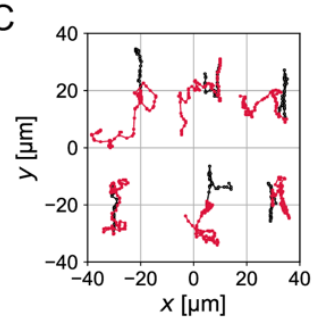

$\mathrm{D}$

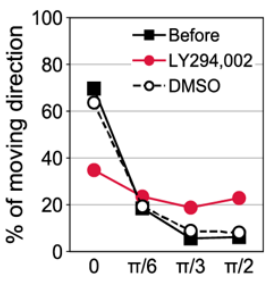

$\mathrm{J}$

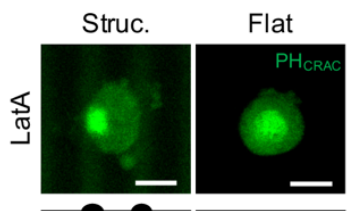

$\mathrm{K}$

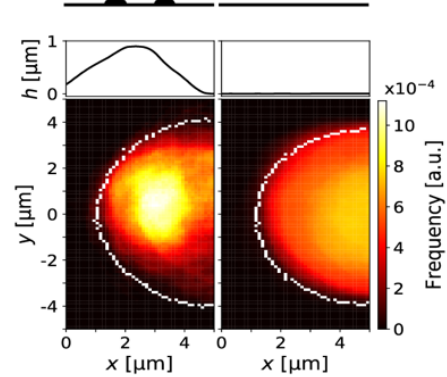

694

695

696

697

698

699

700

701

702

703

704

705

706

707

708

709

710

711

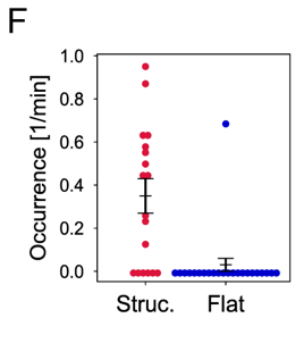

G
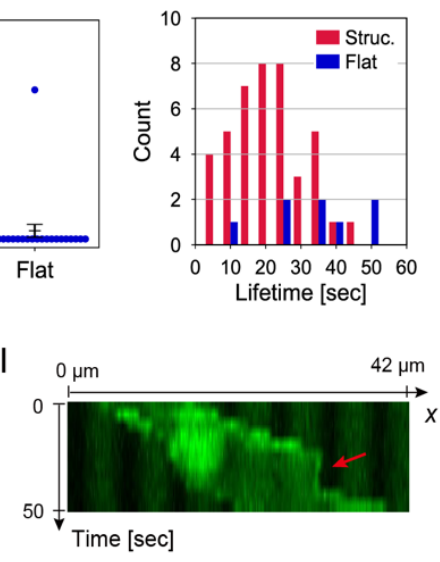

Figure 2. Micro-topographical guidance is PI3K-dependent.

(A) Fraction of patch-positive AX4 cells in aggregation-stage after LY294,002 treatment (mean \pm s.e., $>13$ cells per condition). Averaged for $15 \mathrm{~min}$, and compared in the same field of view before $(t=$ -15 to $0 \mathrm{~min}$ ) and after ( $t=10$ to $25 \mathrm{~min})$ application of the inhibitor $(t=0 \mathrm{~min})$. The measurement was from several field of views in two independent trials per condition.

(B) Merged confocal images (green: GFP-Lifeact fluorescence, red: Alexa594 in the LY294,002 solution, grey: transmitted light, yellow lines: the trajectory of cell centroid). Time in sec; LY294,002 application from a microneedle at $\mathrm{t}=0$. Scale bar, $10 \mu \mathrm{m}$. (C) Cell trajectories from $\mathrm{t}=-5$ to $0 \mathrm{~min}$ (black) and from $\mathrm{t}=0$ to $13 \mathrm{~min}$ (red) . (D) Angular distribution of cell displacement relative to the ridges, before (black solid line; $\mathrm{N}=14$ cells) and after LY294,002 application (red line; $\mathrm{N}=6$ cells). DMSO mock control (black broken line; $\mathrm{N}=8$ cells). (E) Distribution of patch nucleation along the $\mathrm{x}$-axis (upper panel: the ridge z-profile) for aggregation-stage (agg) AX4 cells, vegetative (veg) AX4 and NC4 cells (middle panel). Cell position at the time of patch nucleation (bottom panel, mean \pm s.e., $\mathrm{N}=27$ (AX4, agg), 28 (AX4, veg) and 45 patches (NC4, veg), each dot represents a unique cell). (F) Frequency of ventral F-actin patch nucleation in vegetative NC4 cells (mean \pm s.e., $\mathrm{N}=18$ (Structured) and 23 cells (Flat), each dot presents a unique cell). (G) Lifetime distribution of the Factin patches in vegetative NC4 cells. (H) Lifeact-GFP/NC4 on microridges (green: Lifeact-GFP 
712 fluorescence; MIP from $z=0$ to $3 \mu \mathrm{m}$ ). Time in sec. Scale bar, $10 \mu \mathrm{m}$. (I) A kymograph along

713 the yellow line in $(\mathrm{H})$. The image is enlarged eight times in time-axis. (J) Representative

714 snapshots from confocal images of vegetative NC4 cells expressing PH $_{\text {CRAC-GFP that are treated with }}$

$7153 \mu \mathrm{M}$ LatA on microstructured (left) and non-structured (right) surfaces (green: $\mathrm{PH}_{\mathrm{CRAC}} \mathrm{GFP}$

716 fluorescence; MIP from $z=0$ to $3 \mu \mathrm{m}$, the lower schematic indicates ridge positions). Scale bars, 5

$717 \mu \mathrm{m} . \quad(\mathbf{K})$ The average spatial profile of ventral PHCRAC-GFP fluorescence in LatA-treated cells;

718 microstructured (left, $\mathrm{N}=15$ cells) and non-structured (right, $\mathrm{N}=14$ cells) surfaces. The ridge

719 profile is shown for reference in the upper panel. White borders indicate the average cell contours.

720 See Materials and Methods for details.

721 
A

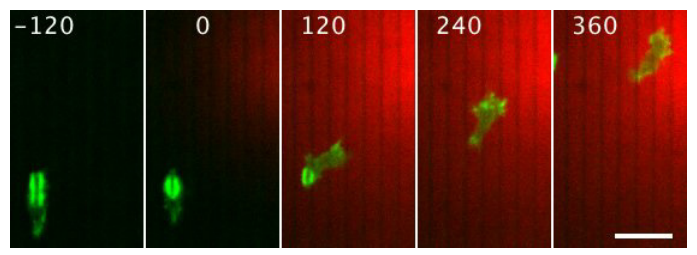

B

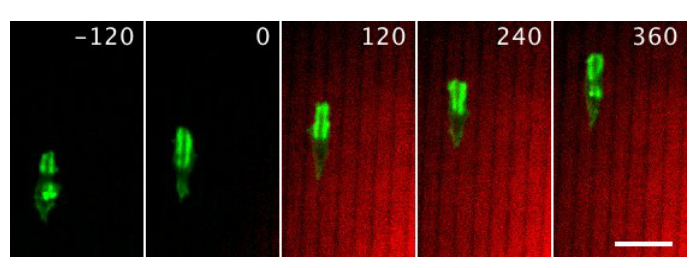

C

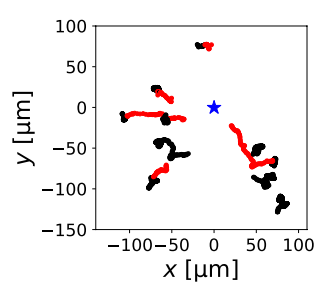

$\mathrm{E}$

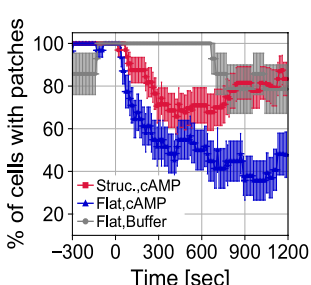

$\mathrm{D}$

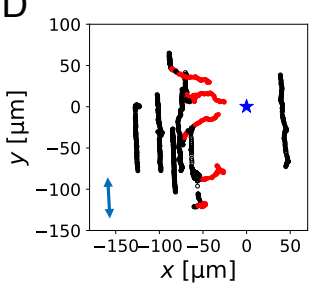

$\mathrm{F}$

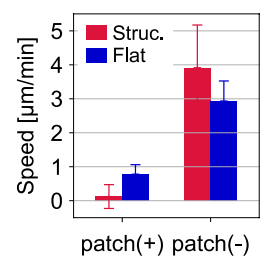

723 Figure 3. Micro-topographical guidance is independent from chemotaxis.

724 (A and B) Confocal timelapse images of cells on the microridges (green: GFP-Lifeact fluorescence,

725 red: Alexa594). Time in sec; cAMP application from a microneedle at $\mathrm{t}=0$. Scale bars, $20 \mu \mathrm{m}$.

726 (C and D) Representative trajectories of cells on non-structured (C) and structured (D) SU-8 surfaces

727 (open: $\mathrm{t}=-5$ to $0 \mathrm{~min}$, closed: $\mathrm{t}=0$ to $15 \mathrm{~min}$, black: patch-positive, red: patch-negative, blue stars:

(E) Fractional change in patch-positive cells after cAMP

729 application (mean \pm s.e., $\mathrm{N}=30$ (structured, cAMP), 30 (non-structured, cAMP) and 14 cells (non-

730 structured, buffer)). (F) Cell migration speeds in the direction of the cAMP source (mean \pm s.e., $\mathrm{N}$

$731=36$ (structured, patch $(+)), 17$ (structured, patch(-)), 32 (non-structured, patch $(+)$ ) and 25 cells (non-

732 structured, patch(-))). Cell migration speed toward cAMP source was determined by quantitating

733 the net centroid displacement towards the microneedle tip for $\Delta t=10 \mathrm{sec}$. 

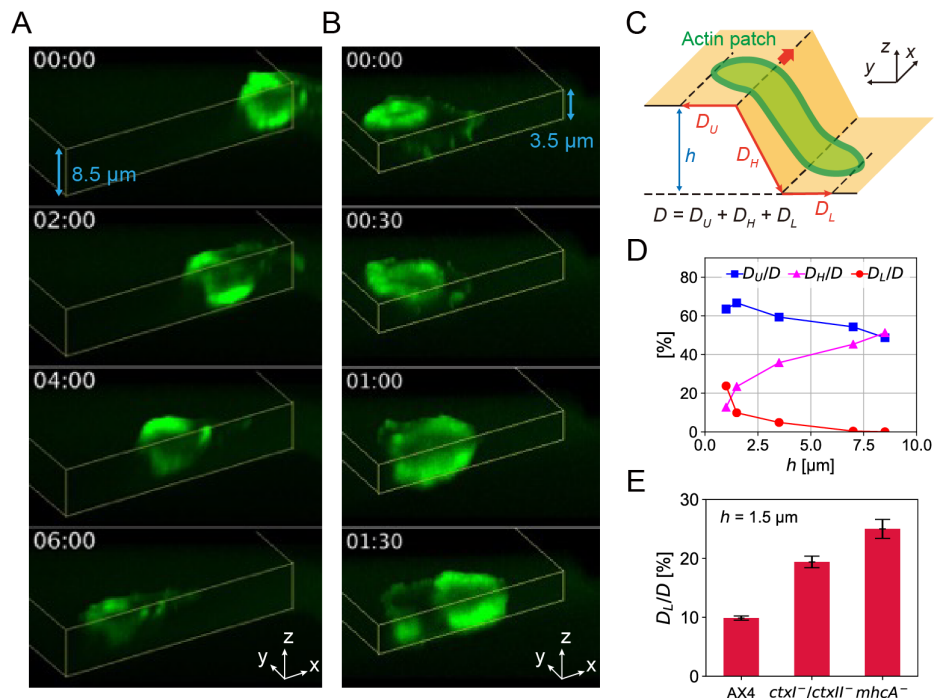

736 Figure 4. The direction of patch propagation is determined by convex and concave surfaces.

737 (A and B) 3-D timelapse confocal images of patch-positive cells on $8.5 \mu \mathrm{m}$ (A) and $3.5 \mu \mathrm{m}$ (B) height

738 plateaus (green: GFP-Lifeact fluorescence; MIP in the direction 60 degrees from the z-axis, yellow

739 lines: contours of plateau surfaces). Time in min:sec. (C) A schematic for parameters $D_{U}, D_{H}, D_{L}$,

$740 D$ and $h$. (D) The height-dependence of $D_{U} / D, D_{H} / D, D_{L} / D$ in AX4 cells. (E) $D_{L} / D$ at $h=1.5 \mu \mathrm{m}$

741 in AX4, ctxI-/ctxII- and $m h c A$ - (mean \pm s.e., $\mathrm{N}=5,9,3$ cells). 
A

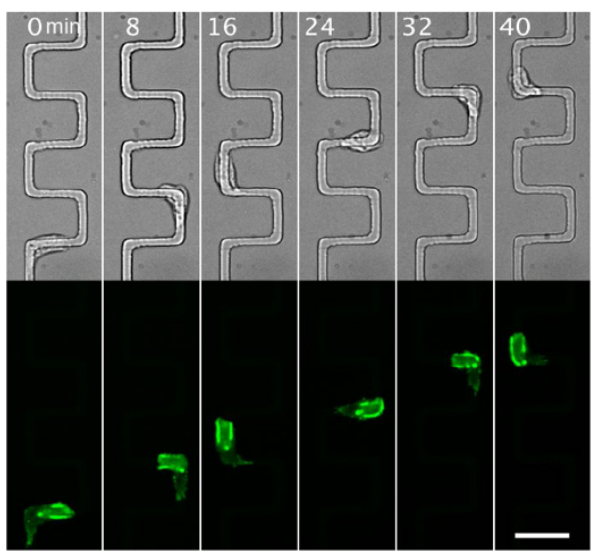

E

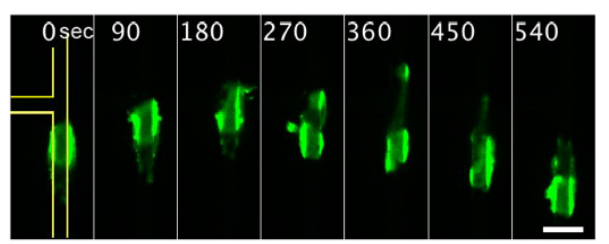

B

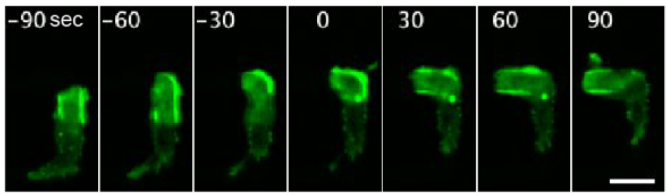

C

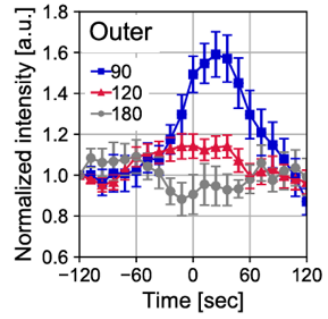

D

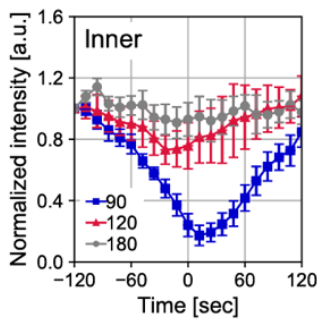

F

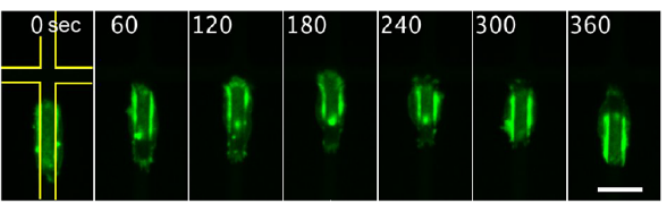

744

Figure 5. F-actin accumulation depends on the corner angle of zig-zag and bifurcating ridges.

Patch and evagination guidance along microridges $(h=1.5 \mu \mathrm{m}$, width $4 \mu \mathrm{m})$ with corners. (A and B) Zig-zag microridges with 90 degrees corners.

(A, upper panel) Transmitted light images.

$(\mathbf{A}$, lower panel and B) GFP-Lifeact fluorescence; MIP from $\mathrm{z}=0$ to $2 \mu \mathrm{m}$. Zoom-up images of turning along the corner (B). (C and D) Change in the GFP-Lifeact intensity along the outer $(\mathbf{C})$ and inner corners (D). Angles are 90 (blue), 120 (red) and 180 (grey) degrees (mean \pm s.e., $\mathrm{N}=16,21,11$ events). $\quad t=0$ is the time when patch centroid reached the corner. $\quad(\mathbf{E}$ and $\mathbf{F})$ Patch reversal at Tjunction $(\mathbf{E})$ and $\mathrm{X}$-junction $(\mathbf{F})$. Yellow lines indicate the ridge contours. Time in $\min (\mathbf{A})$ and $\sec (\mathbf{B}, \mathbf{E}, \mathbf{F}) . \quad$ Scale bars, $20 \mu \mathrm{m}(\mathbf{A})$ and $10 \mu \mathrm{m}(\mathbf{B}, \mathbf{E}, \mathbf{F})$. 
A

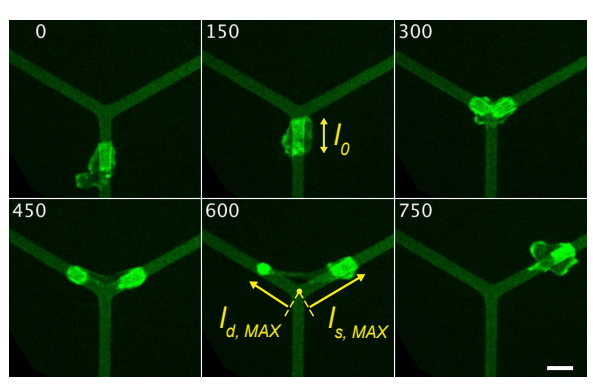

D
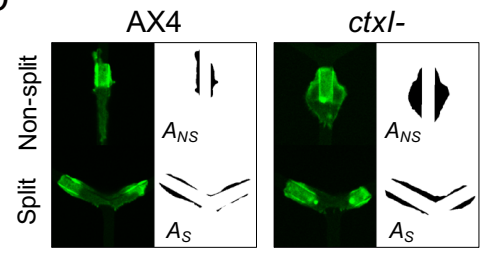

ctxl-/ctxll-
B

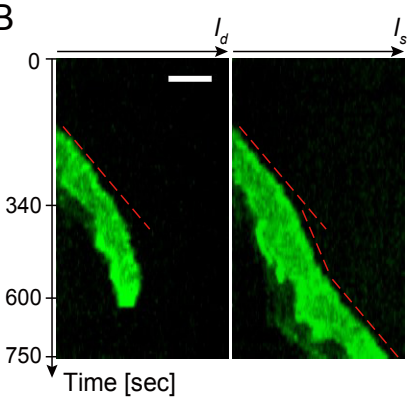

E
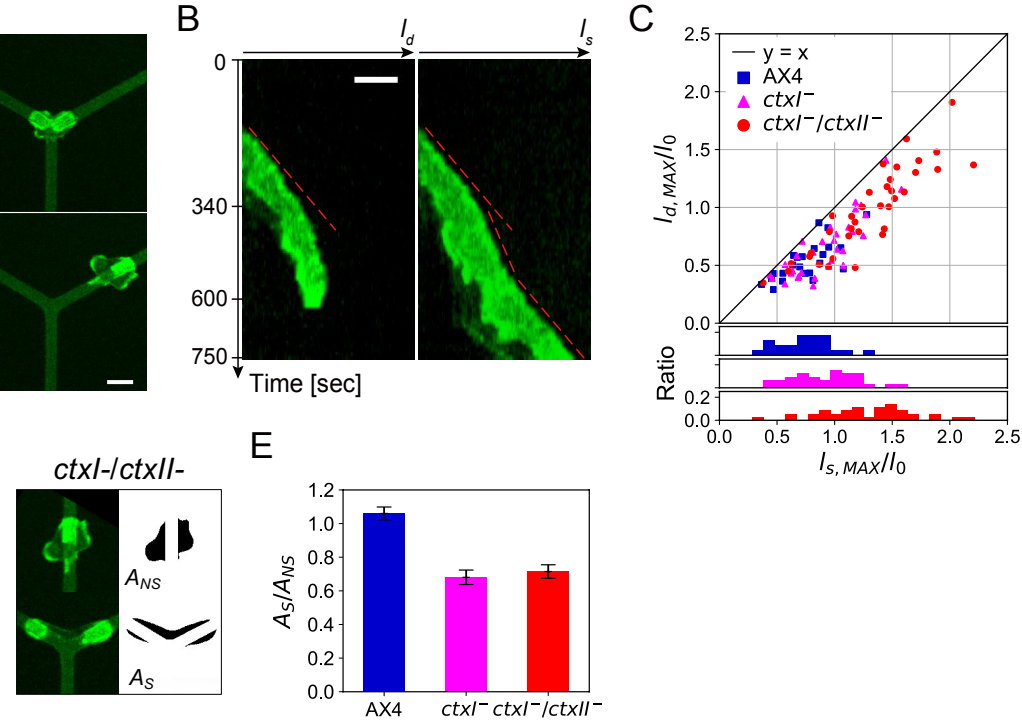

756

757

758

759

760

761

762

763

764

765

766

Figure 6. Membrane tension facilitates confinement of the patches to the ridge.

(A) Time-lapse confocal images of Lifeact-neon/ctxI-/ctxII- at Y-junction. Time in sec. Scale bar, $10 \mu \mathrm{m}$. The ridge is $1.5 \mu \mathrm{m}$ high and $4 \mu \mathrm{m}$ wide. (B) Kymographs taken along each ridge branch in A. Images in $\mathbf{B}$ are enlarged four times in time-axis. Scale bar, $10 \mu \mathrm{m}$. (C) Scatter plots (upper panel) and histograms (lower panles) of maximal elongation rate $l_{s, M A X} / l_{0}$ and $l_{d, M A X} / l_{0}$ in AX4, ctxIand $c t x I-/ c t x I I-(\mathrm{N}=23,32,36$ events). (D) Representative snapshots (left panels, green: GFPLifeact or Lifeact-neon fluorescence) and cell masks outside the ridge (right panels) of AX4, ctxIand $c t x I-/ c t x I I$ - cells with a single patch (upper) and two split patches (lower). (E) Ratio of $A_{S}$ and $A_{N S}$, cell areas outside the ridge during patch splitting and otherwise, in AX4, ctxI- and ctxI-/ctxIIcells (mean \pm s.e., $\mathrm{N}=8,14,17$ events). 


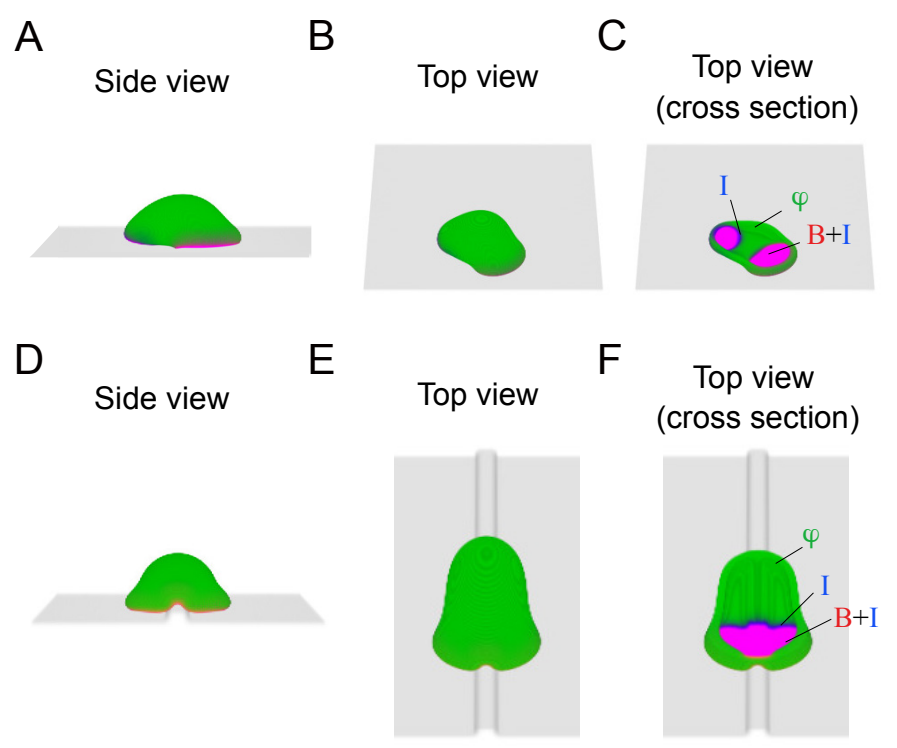

768 Figure 7. Macropinocytic cup formation can capture a microridge.

769 (A-C) Representative snapshots of simulations on a flat substrate. The signaling active patch (red;

$770 A \psi>0$ ), the inhibitor molecule (blue; $I \psi>0$ ) and the membrane (green; $\psi>0$ ) shown as merged

771 RGB images; side view (A), birds-eye view (B), the cross section along the plane parallel to the

772 surface (C). (D-F) Representative snapshots of model simulations with a microridge of height =

$7731.5 \mu \mathrm{m}$ and width $=3.0 \mu \mathrm{m}$. Parameters: $d x=0.2 \mu \mathrm{m}, d t=2 \times 10^{-4} \mathrm{sec}, \varepsilon=1.2 \mu \mathrm{m}$,

$774 \quad M_{V}=5.0, \tau=10.0 \mathrm{nN} \cdot \mathrm{sec} / \mu \mathrm{m}^{3}, F=2.6 \mathrm{nN} / \mu \mathrm{m}^{2}, \eta=0.7 \mathrm{nN} / \mu \mathrm{m}, \beta=100.0, \theta=0.105$,

$775 k_{1}=0.05, k_{2}=0.5, a_{\mathrm{t}}=1.6, D_{\mathrm{a}}=0.17, D_{\mathrm{i}}=0.1$ for $(\mathbf{A}-\mathbf{C})$ and $D_{\mathrm{i}}=0.13$ for $(\mathbf{D}-\mathbf{F}), K_{1}=$ $7760.05, K_{2}=0.04$. 


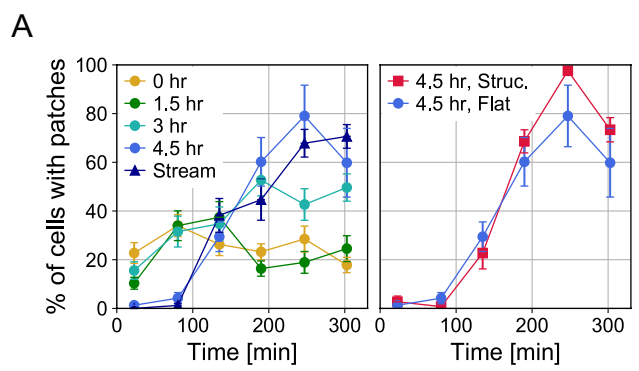

B

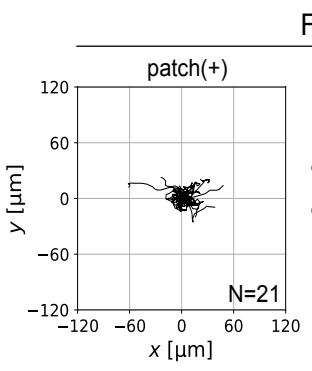

Flat

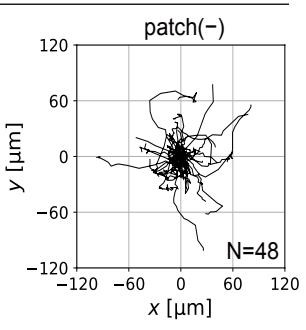

C
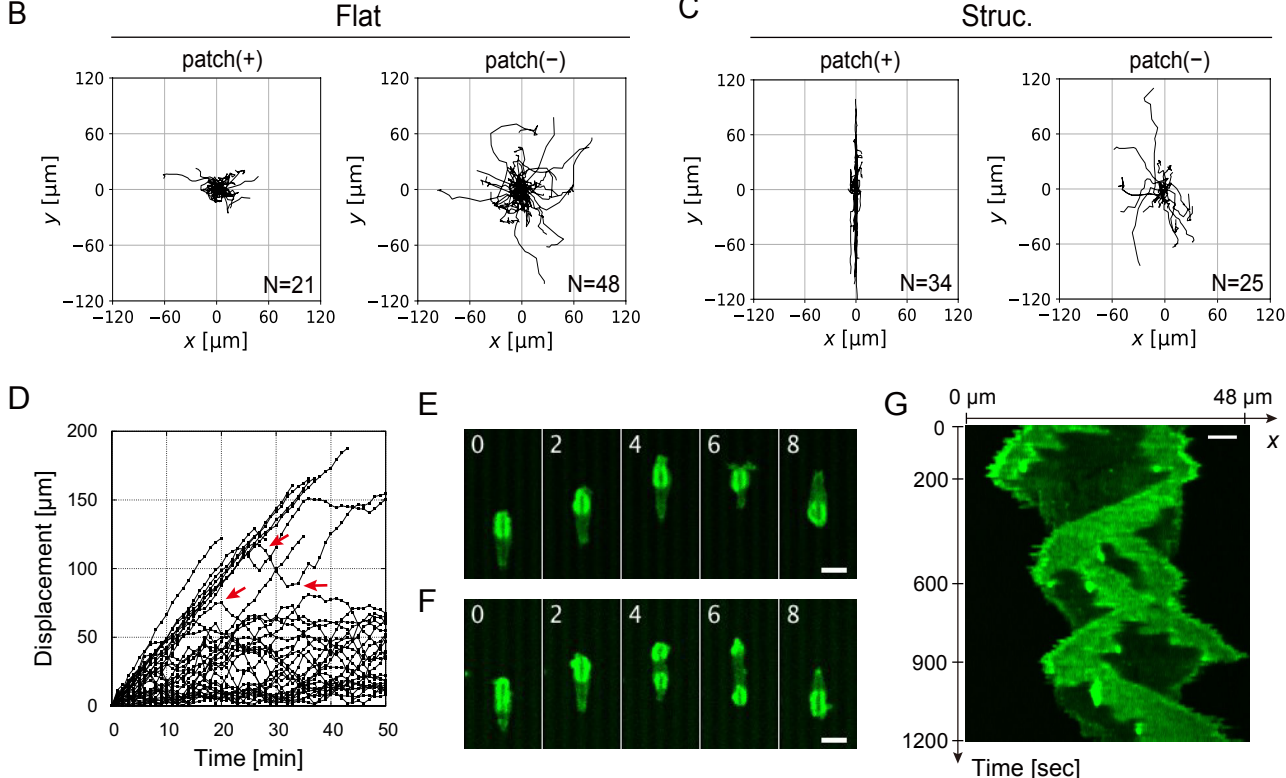

E
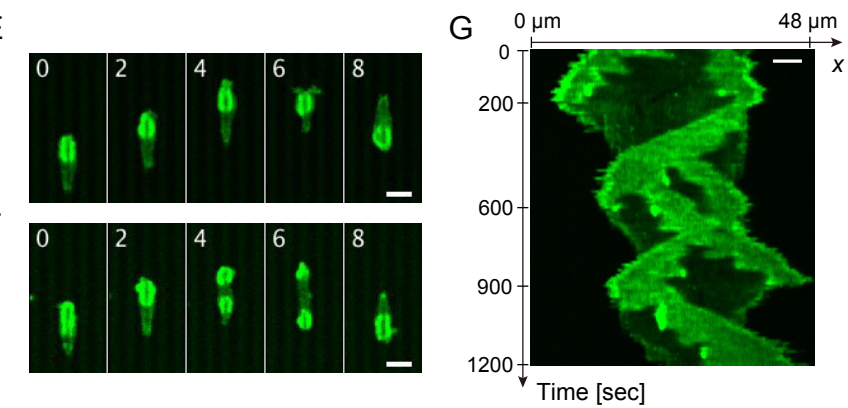

Figure S1. Patch nucleation and migration on flat and micro-structured SU-8 surfaces.

780 (A) Change in the percentage of patch-positive AX4 cells placed on the SU-8 surface over the course of $6 \mathrm{hrs}$ from the time of plating (mean \pm s.e., $>17$ cells per condition). (A, left panel) Cells on the nonstructured surface that were differentiated with cAMP pulsing $(0,1.5,3.0,4.5 \mathrm{hr})$ or collected from aggregation streams on agar (stream). (A, right panel) Cells on microstructured and nonstructured surfaces that were differentiated with cAMP pulsing for $4.5 \mathrm{hr}$. (B and C) Trajectories for 20 min of patch-positive or negative cells on non-structured (B) and microstructured (C) surfaces where $1 \mu \mathrm{m}$ high and $3 \mu \mathrm{m}$ wide ridges were placed at an interval of $3 \mu \mathrm{m} . \quad \mathrm{N}=21$ (Flat, patch $(+)$ ), 48 (Flat, patch $(-)), 34$ (Structured, patch $(+))$ and 25 cells (Structured, patch $(-)$ ).

(D) Time

788 courses of cell displacement along straight ridges in the presence of patches $(\mathrm{N}=36$ cells). Turnings 789 (red arrows). (E and F) Confocal timelapse images (green: GFP-Lifeact fluorescence; $z=0$ ). Time 790 in min. Scale bars, $10 \mu \mathrm{m}$. (G) A kymograph taken from a cell on the ridge with small net 791 displacement. Scale bar, $5 \mu \mathrm{m}$. 

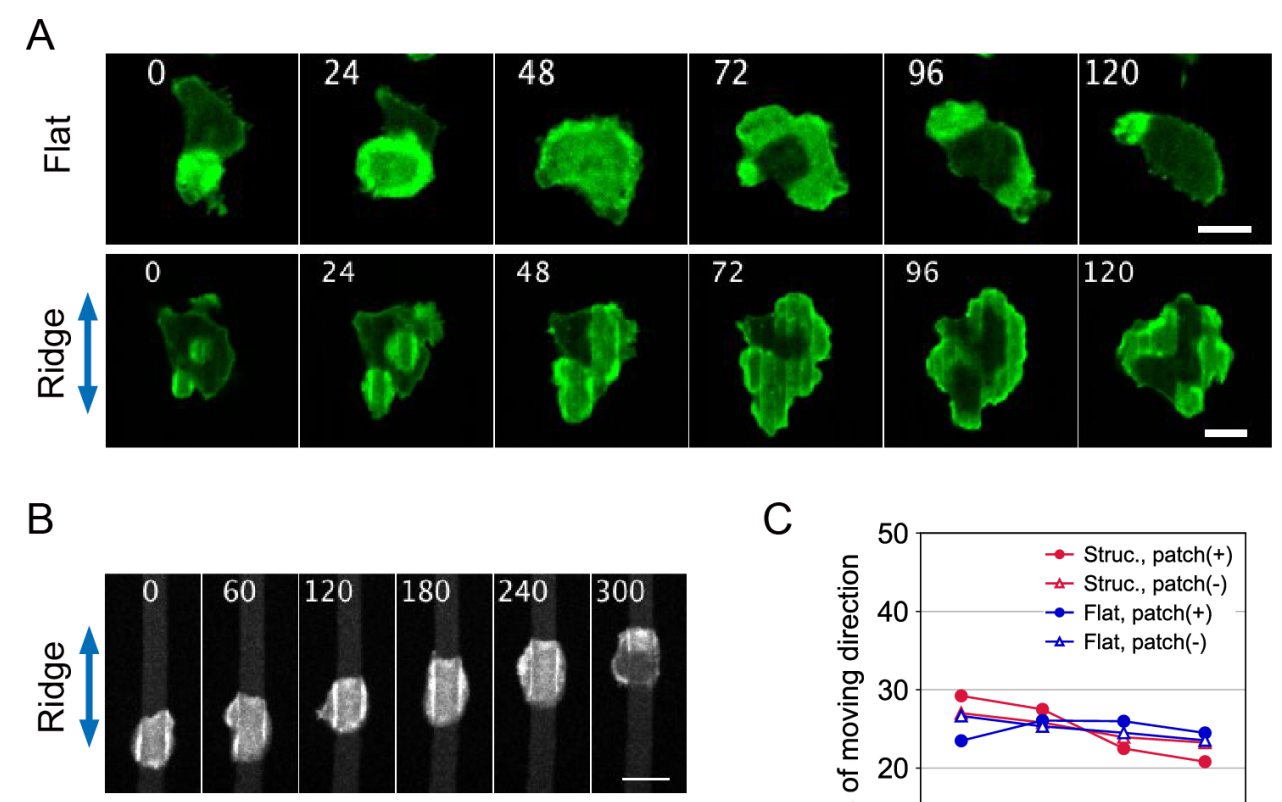

C

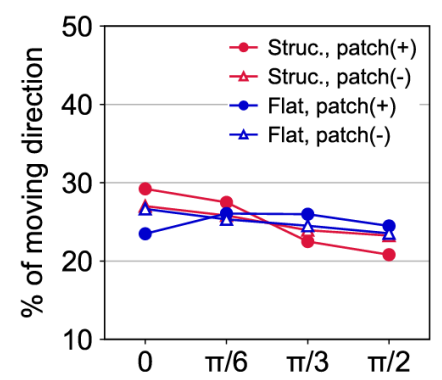

793 Figure S2. Guidance of the ventral F-actin patches and membrane evagination along a 794 microridge in the growth-stage AX4 cells.

795 (A and B) Time-lapse confocal images of growth-stage AX4 cells taken near the substrate. A cell 796 on the non-structured (A, upper panel) and microstructured (A, lower panel) SU-8 surface. Ridges 797 are $1 \mu \mathrm{m}$ high and $3 \mu \mathrm{m}$ wide and placed parallely at an even $3 \mu \mathrm{m}$ spacing (green: GFP-Lifeact 798 fluorescence; A, upper panel: $z=0$, A, lower panel: MIP from $z=0$ to $2 \mu \mathrm{m}$ ). (B) Time-lapase 799 confocal images of a GFP-Lifeact/AX4 cell on a $1.5 \mu \mathrm{m}$ high and $5 \mu \mathrm{m}$ wide microridge placed 800 parallelly at $10 \mu \mathrm{m}$ spacing (grey: Lifeact-mRFPmars fluorecence, MIP from $z=0$ to $2 \mu \mathrm{m}$ ). Time 801 in sec. Scale bars, $10 \mu \mathrm{m}$. (C) Angular distribution of cell displacement relative to the ridge 802 orientation. 


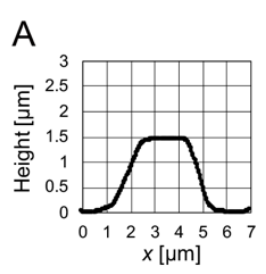

B
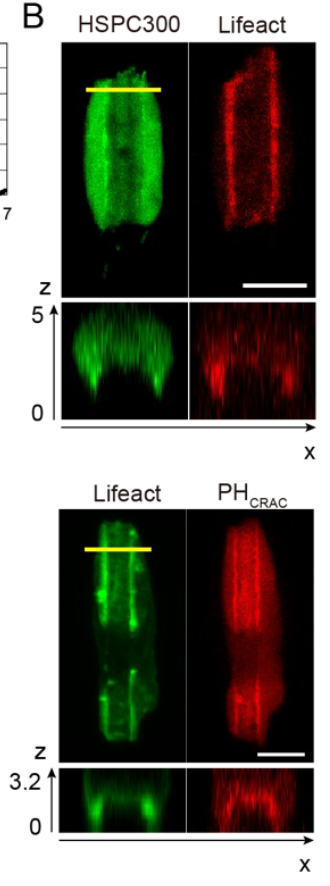
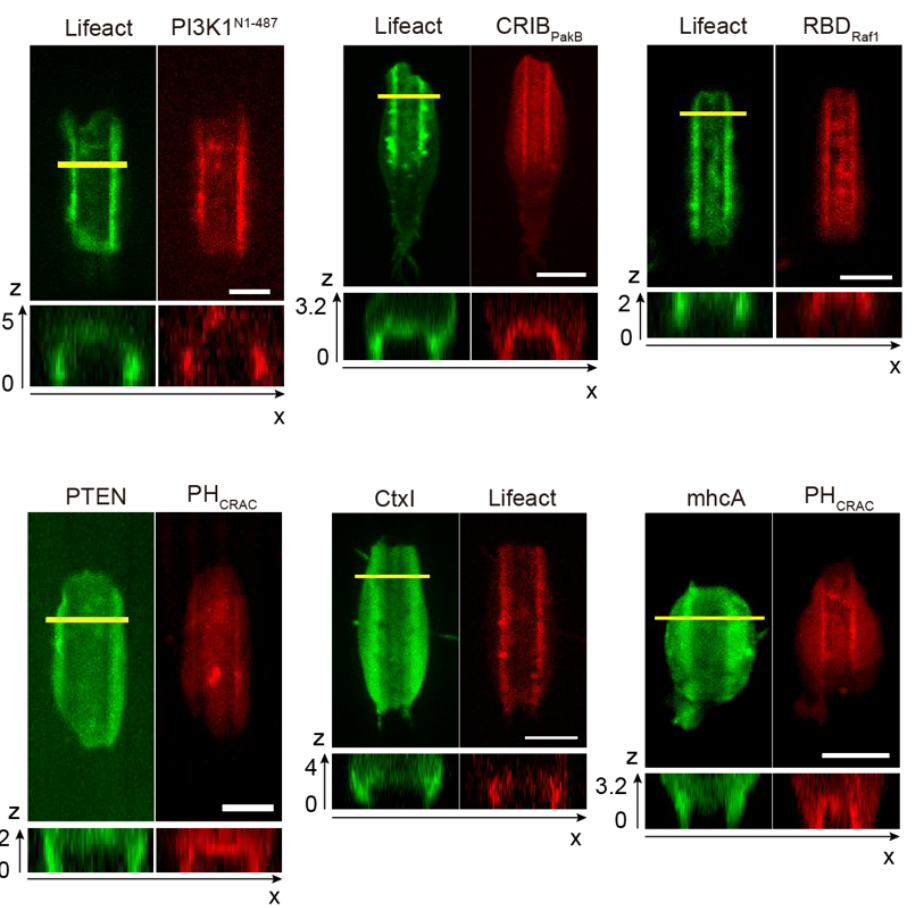

804

805 Figure S3. Localization of the macropinocytic/phagocytic patch components along the 806 microridge.

807 (A) The profile of an etched glass surface. (B) Confocal images of patch-positive cells that co-

808 express GFP- and RFP- fluorescent probes (from left to right and top to bottom): SCAR complex/F-

809 actin (HSPC300-GFP/Lifeact-mRFPmars/AX4), F-actin/PI3K1 (GFP-Lifeact/PI3K1Nterm-

810 RFPmars/AX4), F-actin/Rac-GTP (GFP-Lifeact/CRIBPakB-mRFP1/AX4), F-actin/Ras-GTP (Lifeact-

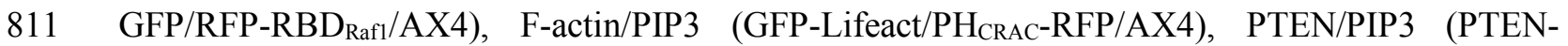

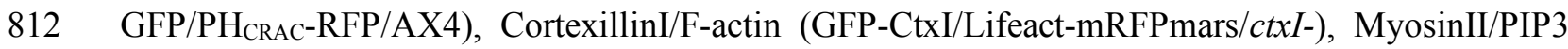

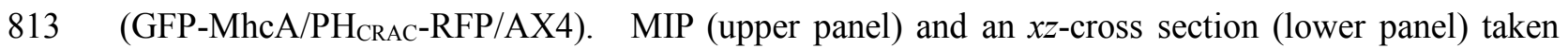

814 along the yellow line in the upper panel. Scale bars are $5 \mu \mathrm{m}$ and the $z$-axis is in the unit of $\mu \mathrm{m}$. 
A

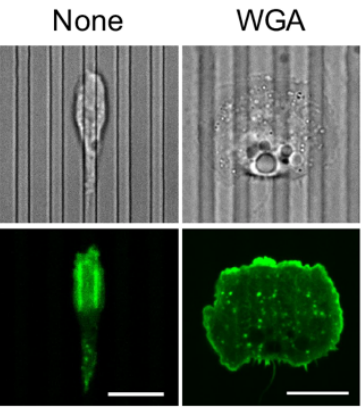

B

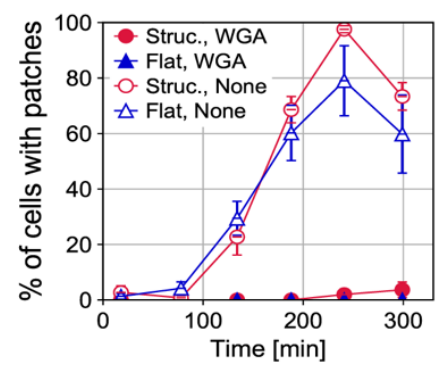

C

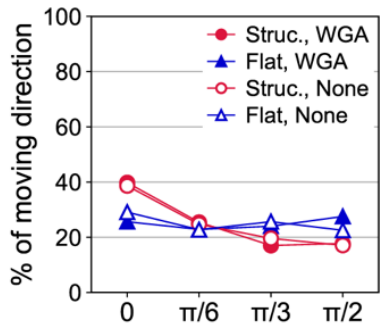

816

\section{Figure S4. Adhesive surface suppresses ventral F-actin patches.}

818 (A) Aggregation-stage GFP-Lifeact/AX4 plated on the lectin WGA-coated structured substrates 819 (upper: transmitted-light, lower: GFP-Lifeact fluorescence). Microridges are $1 \mu \mathrm{m}$ high and $3 \mu \mathrm{m}$

820 wide. (B) Time series of the percentage of patch-positive cells on the coated substrates (mean \pm s.e., $821>18$ cells per condition). Non-coat data are shown for comparison (duplicated from Fig. S1A, right 822 panel). Cells are plated at time $0 \mathrm{~min}$. (C) Angular distribution of migratory directions of cells on 823 the coated substrates. Non-coated data are shown for comparison (duplicated from Fig. 1E, patch(-)). $824 \mathrm{~N}=173$ (Structured, WGA), 160 (Flat, WGA), 21 (Structured, Non-coated) and 36 cells (Flat, Non825 coated).

826 
A

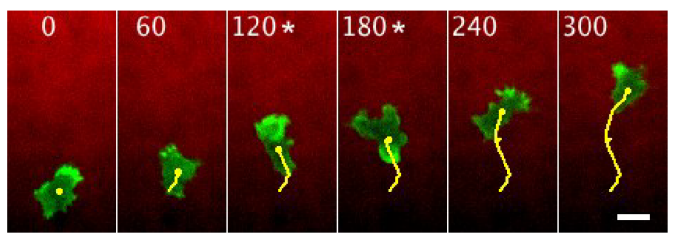

C

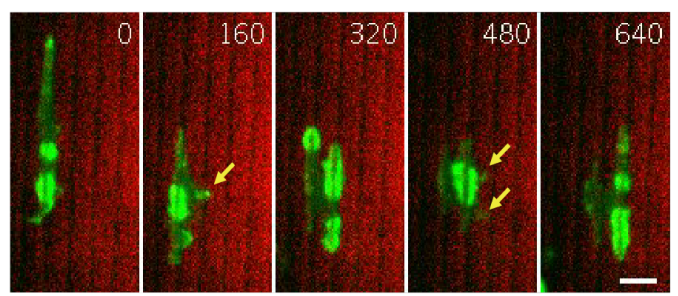

B

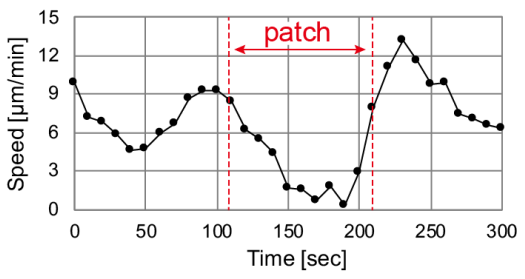

828 Figure S5. AX4 cell movement under an extracellular cAMP gradient is interfered by ventral 829 actin patches.

830 Aggregation-stage GFP-Lifeact/AX4 stimulated with 100 nM cAMP (green: GFP-Lifeact, red:

831 Alexa594). Alea594 is included in the cAMP source as an indicator. (A) Representative time-

832 lapse images on a flat SU-8 surface. Trajectory of the cell centroid (yellow lines). The asterisk

833 indicates the presence of a patch. (B) Cell migration speed for the time sequence shown in A. (C)

834 Representative time-lapse images of GFP-Lifeact/AX4 cells on the structured substrate. Small 835 projections toward the cAMP source (yellow arrows). Time in sec. Scale bars, $10 \mu \mathrm{m}$.

836 

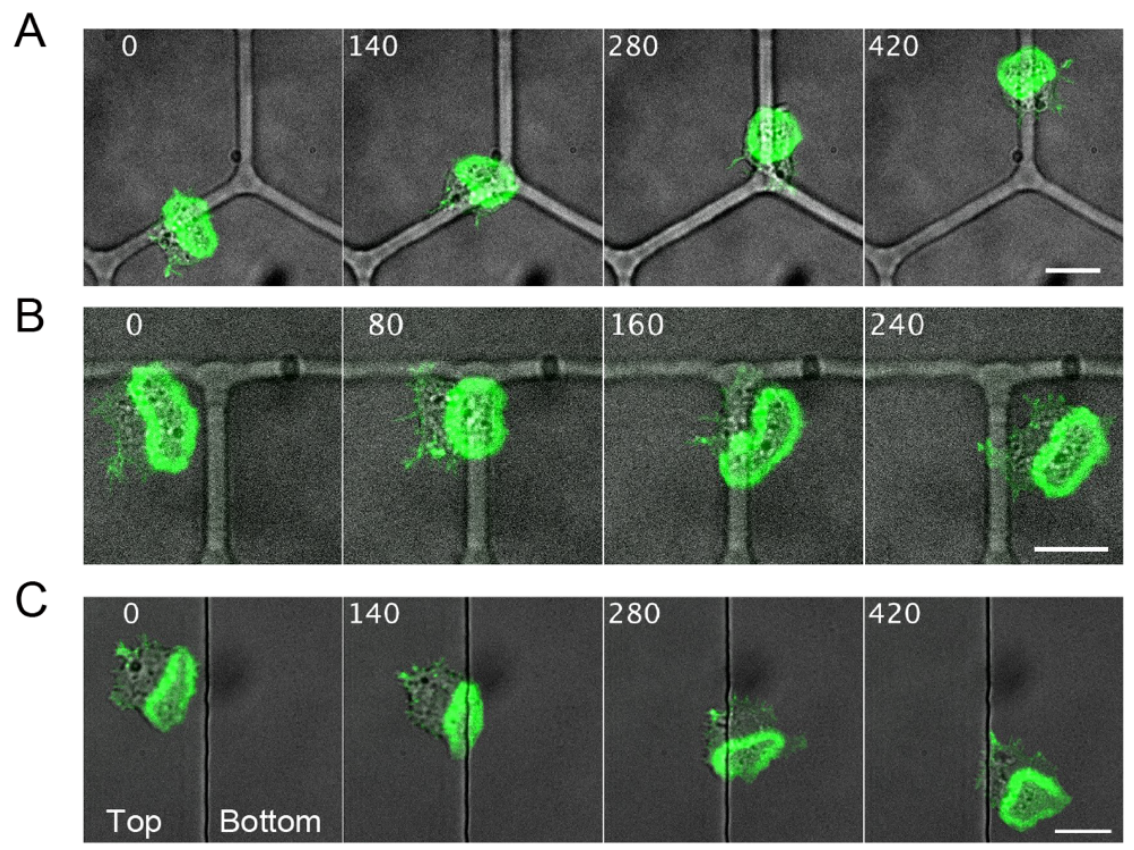

838 Figure S6. Myosin II-null cells are less confined to micro-structures.

839 Lifeact-neon $/ m h c A$ - cells taken near the substrate (green: Lifeact-neon fluorescence, grey:

840 transmitted-light). The ridge is $1.5 \mu \mathrm{m}$ high and $4 \mu \mathrm{m}$ wide ( $\mathbf{A}$ and $\mathbf{B}$ ) and the edge of a plateau

841 surface is $1.5 \mu \mathrm{m}$ in height $(\mathbf{C})$. Images in $\mathbf{C}$ are MIP from $z=0$ to $2 \mu \mathrm{m}$. Time in sec. Scale

842 bars, $10 \mu \mathrm{m}$. 
A

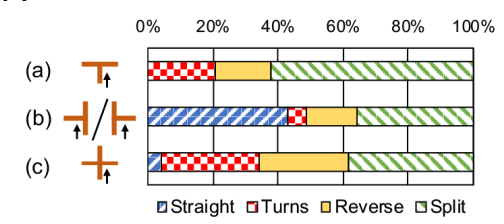

B

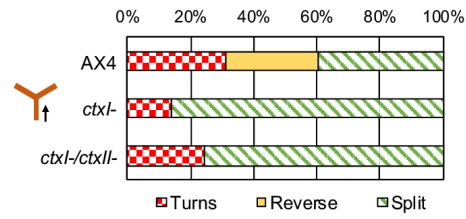

C

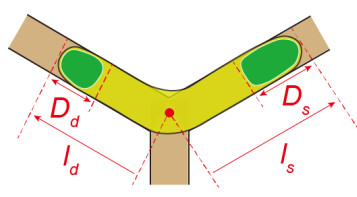

$\mathrm{D}$

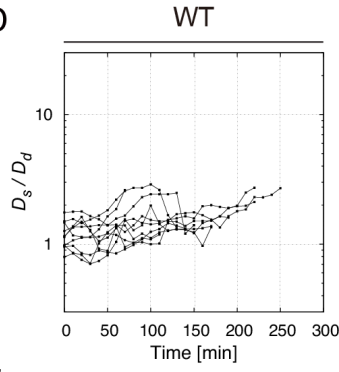

E

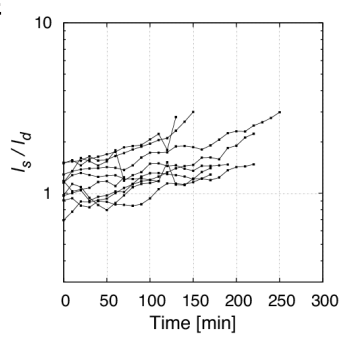

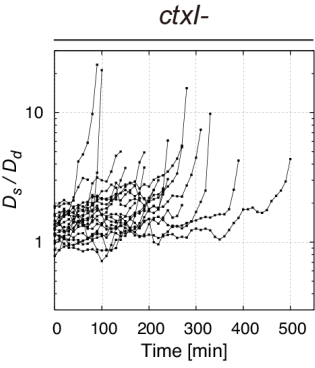
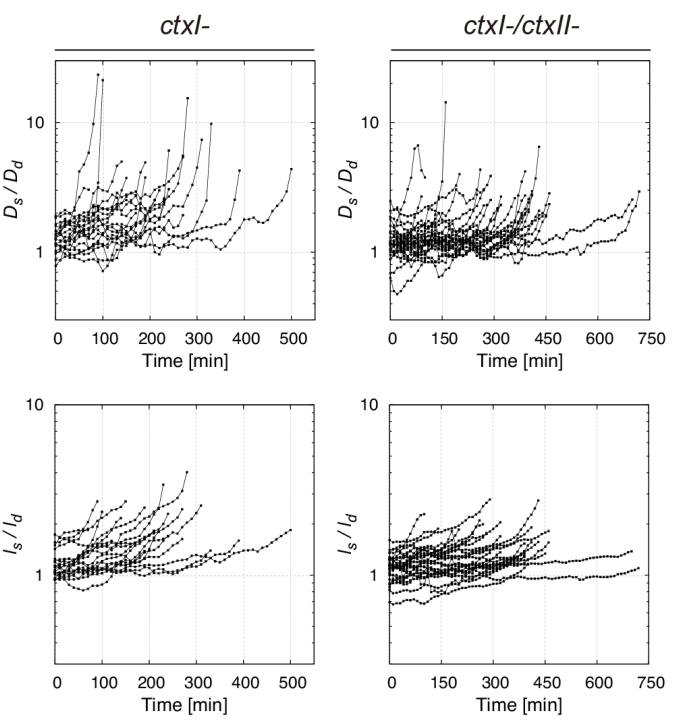

844

845

846 (A) Distribution of the patch behaviors at T- and X-junctions (a-c). $\mathrm{N}=29$ (a), 84 (b) and 76 (c)

847 events. (B) Distribution of the patch behaviors at Y-junction. $\mathrm{N}=58$ (AX4), 43 (ctxI-) and 57

848 (ctxI-/ctxII-) events. (C) A schematic of patch diameters $D_{s}$ and $D_{d}$ and the distances from the

849 junction point to the bifurcating cell edge $I_{s}, I_{d}$. Subscripts ' $s$ ' and ' $d$ ' signify patches that 'survived'

850 or 'disappeared', respectively. (D and E) Time course of $D_{s} / D_{d}$ and $l_{s} / l_{d}$ at Y-junction in AX4 $(\mathrm{N}=$

85110 events), ctxI- $(\mathrm{N}=20$ events $)$ and $c t x I-/ c t x I I-(\mathrm{N}=28$ events $)$. 
A

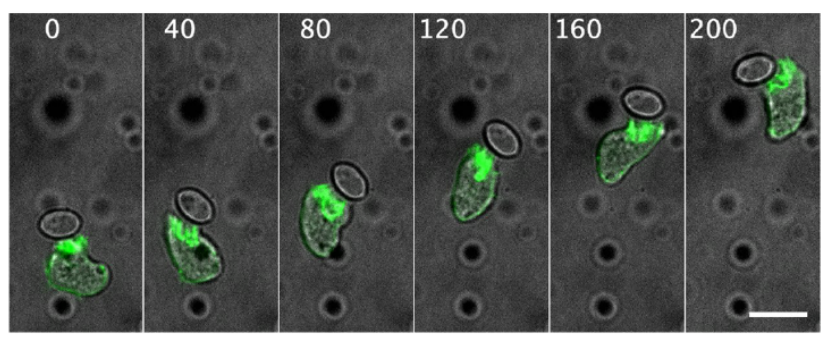

B

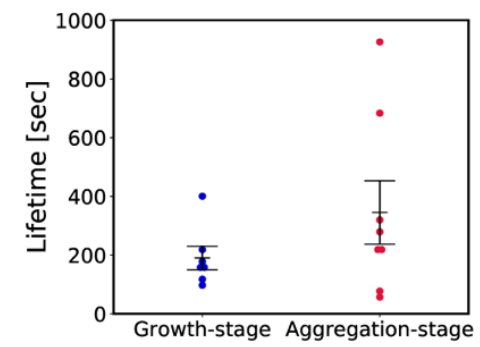

853

854 Figure S8. Directed migration towards an attached yeast particle.

855 (A) Time-lapse images of aggregation-stage Lifeact-neon/AX2 cell in contact with a yeast particle

856 (grey: transmitted light, green: Lifeact-neon fluorescence). Time in sec. Scale bar, $10 \mu \mathrm{m}$.

(B)

857 Lifetime of actin patches at the interface between the yeast particle and Dictyostelium in growth-stage

858 and aggregation-stage cells (mean \pm s.e.; $N=7,8$ patches, each dot represents a unique cell). 
860

861

862

863

864

865

866

867

868

869

870

871

872

873

874

875

876

877

878

879

880

881

882

883

884

885

886

887

888

889

890

891

\section{Movie legends}

\section{Movie S1. Propagating ventral F-actin patch in aggregation-stage Dictyostelium AX4.}

Time-lapse confocal images of GFP-Lifeact/AX4 cells on a non-structured SU-8 surface. Images were acquired every $2 \mathrm{sec}$. Time in sec. Scale bar is $10 \mu \mathrm{m}$.

\section{Movie S2. Topography-guidance of the ventral F-actin patch and membrane evagination in} aggregation-stage Dictyostelium AX4.

Time-lapse confocal images of GFP-Lifeact/AX4 on a microstructured SU-8 surface (green: GFPLifeact; maximum intensity projection from $\mathrm{z}=0$ to $2 \mu \mathrm{m}$ taken at an interval of $0.5 \mu \mathrm{m}$ from the SU-8 surface). Images were acquired every $10 \mathrm{sec}$. The ridge is $1 \mu \mathrm{m}$ high and $3 \mu \mathrm{m}$ wide placed at an interval of $3 \mu \mathrm{m}$. Time in sec. Scale bar is $10 \mu \mathrm{m}$.

\section{Movie S3. Topography-dependence of PIP3 signaling patch in LatA-treated NC4 cells.}

Time-lapse confocal images of vegetative $\mathrm{PH}_{\mathrm{CRAC}}-\mathrm{GFP} / \mathrm{NC} 4$ cells treated with $3 \mu \mathrm{M}$ Latrunculin A on microstructured (left) and non-structured (right) surfaces (green: PH $_{\text {CRAC-GFP fluorescence; MIP }}$ from $z=0$ to $3 \mu \mathrm{m}$ every $0.5 \mu \mathrm{m}$, the lower schematic indicates ridge positions). Images were acquired every $10 \mathrm{sec}$. The ridge is $1 \mu \mathrm{m}$ high and $3 \mu \mathrm{m}$ wide placed in parallel at an interval of 3 $\mu \mathrm{m}$. Time in sec. Scale bars are $5 \mu \mathrm{m}$.

\section{Movie S4. Extinction of F-actin patch and chemotaxis to extracellular cAMP.}

Time-lapase confocal images of GFP-Lifeact/AX4 on a microstructured SU-8 surface (green: GFPLifeact; z-slice near the SU-8 surface, red: Alexa594, an indicator of cAMP). Transient F-actin patches (left) and a persistent patch (right). Images were acquired every $10 \mathrm{sec}$. cAMP was applied at $t=0 \mathrm{sec}$ from a microneedle. Time in sec. Scale bars $20 \mu \mathrm{m}$.

\section{Movie S5. Guidance of F-actin patch and membrane evagination along the zig-zag patterns.}

Timelapse images of GFP-Lifeact/AX4 cells on a microridge with alternating \pm 90 degrees corners. Transmitted light (left) and confocal (right). Grey: transmitted light. Green: GFP-Lifeact; maximum intensity projection from $\mathrm{z}=0$ to $2 \mu \mathrm{m}$ taken at an interval of $0.5 \mu \mathrm{m}$ from the SU-8 surface. The ridge is $1.5 \mu \mathrm{m}$ high and $4 \mu \mathrm{m}$ wide. Images were acquired every $6 \mathrm{sec}$. Time in min. Scale bar $10 \mu \mathrm{m}$. 
893 Movie S6. Turning of the ventral F-actin patch and the leading edge at $\mathbf{X}$-junctions.

894 Confocal timelapse images of GFP-Lifeact on the microstructured SU-8 surface (green: maximum 895 intensity projection from $\mathrm{z}=0$ to $2 \mu \mathrm{m}$ taken at an interval of $0.5 \mu \mathrm{m}$ from the SU- 8 surface). Images 896 were acquired every $12 \mathrm{sec}$. The ridge is $1.5 \mu \mathrm{m}$ high and $4 \mu \mathrm{m}$ wide. Two X-junctions appear 897 at the top and bottom of the frame. Time in sec. Scale bar $5 \mu \mathrm{m}$.

900 Confocal timelapse images of a Lifeact-neon/ctxI-/ctxII- cells on a microstructured SU-8 surface

901 (green: z-slice near the SU-8 surface). Images were acquired every $10 \mathrm{sec}$. The ridges are $1.5 \mu \mathrm{m}$

902 high and $4 \mu \mathrm{m}$ wide and connected to form Y-junctions. Time in sec. Scale bar is $10 \mu \mathrm{m}$.

903

904

Movie S8. Model simulations of the patch dynamics and the resulting plasma membrane

905 deformation.

906 Timelapse of the representative data shown in Fig. 7D-F for a flat surface (left panels) and for a

907 microridge (right panel). Periodic boundary conditions are employed at $x=0,40 \mu \mathrm{m}$ and $y=0,60$ $908 \mu \mathrm{m}$.

909

910

911

\section{References}

912

913

Amara A, Mercer J. 2015. Viral apoptotic mimicry. Nat Rev Microbiol 13:461-9. doi:10.1038/nrmicro3469

Asano Y, Nagasaki A, Uyeda TQP. 2008. Correlated waves of actin filaments and PIP3 in Dictyostelium cells. Cell Motil Cytoskel 65:923-934. doi:10.1002/cm.20314

Barger SR, Reilly NS, Shutova MS, Li Q, Maiuri P, Heddleston JM, Mooseker MS, Flavell RA, Svitkina T, Oakes PW, Krendel M, Gauthier NC. 2019. Membrane-cytoskeletal crosstalk 
920

921

922

923

924

925

926

927

928

929

930

931

932

933

934

935

936

937

938

939

940

941

942

943

944

mediated by myosin-I regulates adhesion turnover during phagocytosis. Nat Commun 10:1249. doi:10.1038/s41467-019-09104-1

Bloomfield G, Traynor D, Sander SP, Veltman DM, Pachebat JA, Kay RR. 2015. Neurofibromin controls macropinocytosis and phagocytosis in Dictyostelium. Elife 4. doi:10.7554/elife.04940

Bretschneider T, Anderson K, Ecke M, Müller-Taubenberger A, Schroth-Diez B, IshikawaAnkerhold HC, Gerisch G. 2009. The three-dimensional dynamics of actin waves, a model of cytoskeletal self-organization. Biophys J 96:2888-2900. doi:10.1016/j.bpj.2008.12.3942

Bretschneider T, Diez S, Anderson K, Heuser J, Clarke M, Müller-Taubenberger A, Köhler J, Gerisch G. 2004. Dynamic Actin Patterns and Arp2/3 Assembly at the Substrate-Attached Surface of Motile Cells. Curr Biol 14:1-10. doi:10.1016/j.cub.2003.12.005

Brzeska H, Koech H, Pridham KJ, Korn ED, Titus MA. 2016. Selective localization of myosin-I proteins in macropinosomes and actin waves. Cytoskeleton 73:68 82. doi:10.1002/cm.21275

Brzeska H, Pridham K, Chery G, Titus MA, Korn ED. 2014. The association of myosin IB with actin waves in dictyostelium requires both the plasma membrane-binding site and actin-binding region in the myosin tail. Plos One 9:e94306. doi:10.1371/journal.pone.0094306

Buczynski G, Grove B, Nomura A, Kleve M, Bush J, Firtel RA, Cardelli J. 1997. Inactivation of Two Dictyostelium discoideum Genes, DdPIK1 and DdPIK2, Encoding Proteins Related to Mammalian Phosphatidylinositide 3-kinases, Results in Defects in Endocytosis, Lysosome to Postlysosome Transport, and Actin Cytoskeleton Organization. J Cell Biol 136:1271-1286. doi:10.1083/jcb.136.6.1271

Case LB, Waterman CM. 2015. Integration of actin dynamics and cell adhesion by a threedimensional, mechanosensitive molecular clutch. Nature Cell Biology 17. doi:10.1038/ncb3191

Case LB, Waterman CM. 2011. Adhesive F-actin waves: a novel integrin-mediated adhesion complex coupled to ventral actin polymerization. Plos One 6:e26631. doi:10.1371/journal.pone.0026631 
945 Champion JA, Mitragotri S. 2006. Role of target geometry in phagocytosis. P Natl Acad Sci Usa

$946 \quad$ 103:4930 4934. doi:10.1073/pnas.0600997103

947 Chen C-L, Wang Y, Sesaki H, Iijima M. 2012. Myosin I links PIP3 signaling to remodeling of the

$948 \quad$ actin cytoskeleton in chemotaxis. Sci Signal 5:ra10. doi:10.1126/scisignal.2002446

949 Clarke M, Engel U, Giorgione J, Müller-Taubenberger A, Prassler J, Veltman D, Gerisch G. 2010.

950 Curvature recognition and force generation in phagocytosis. Bmc Biol 8:154. doi:10.1186/1741-

$951 \quad 7007-8-154$

952 Cox D, Tseng C-C, Bjekic G, Greenberg S. 1999. A Requirement for Phosphatidylinositol 3-Kinase

953 in Pseudopod Extension. J Biol Chem 274:1240-1247. doi:10.1074/jbc.274.3.1240

954 Driscoll MK, Sun X, Guven C, Fourkas JT, Losert W. 2014. Cellular Contact Guidance through

955 Dynamic Sensing of Nanotopography. Acs Nano 8:3546-3555. doi:10.1021/nn406637c

956

957

958

959

960

961

962

963

964

965

966

967

Dunn GA. 1983. Leukocyte Locomotion and Chemotaxis. Agents and Actions Supplements 12:1433. doi:10.1007/978-3-0348-9352-7_1

Faix J, Weber I, Mintert U, Köhler J, Lottspeich F, Marriott G. 2001. Recruitment of cortexillin into the cleavage furrow is controlled by Rac1 and IQGAP-related proteins. Embo J 20:3705 3715. doi:10.1093/emboj/20.14.3705

Fey P, Stephens S, Titus MA, Chisholm RL. 2002. SadA, a novel adhesion receptor in Dictyostelium. J Cell Biology 159:1109-1119. doi:10.1083/jcb.200206067

Flemming S, Font F, Alonso S, Beta C. 2020. How cortical waves drive fission of motile cells. Proc National Acad Sci 117:6330-6338. doi:10.1073/pnas.1912428117

Franco D, Klingauf M, Bednarzik M, Cecchini M, Kurtcuoglu V, Gobrecht J, Poulikakos D, Ferrari A. 2011. Control of initial endothelial spreading by topographic activation of focal adhesion kinase. Soft Matter 7:7313-7324. doi:10.1039/c1sm05191a 
968 Friedl P, Alexander S. 2011. Cancer invasion and the microenvironment: plasticity and reciprocity. Cell 147:992-1009. doi:10.1016/j.cell.2011.11.016 proteins control surface expression and stability of SibA adhesion molecules in Dictyostelium. Mol Biol Cell 23:679-686. doi:10.1091/mbc.e11-04-0338

Fujimori T, Nakajima A, Shimada N, Sawai S. 2019. Tissue self-organization based on collective cell migration by contact activation of locomotion and chemotaxis. Proc National Acad Sci 116:4291 4296. doi:10.1073/pnas.1815063116

Fukushima S, Matsuoka S, Ueda M. 2019. Excitable dynamics of Ras triggers spontaneous symmetry breaking of PIP3 signaling in motile cells. J Cell Sci 132:jcs224121. doi: $10.1242 /$ jcs. 224121

Galic M, Jeong S, Tsai F-C, Joubert L-M, Wu YI, Hahn KM, Cui Y, Meyer T. 2012. External push and internal pull forces recruit curvature-sensing N-BAR domain proteins to the plasma membrane. Nat Cell Biol 14:874 881. doi:10.1038/ncb2533

Gallop JL, Walrant A, Cantley LC, Kirschner MW. 2013. Phosphoinositides and membrane curvature switch the mode of actin polymerization via selective recruitment of toca-1 and Snx9. Proc National Acad Sci 110:7193-7198. doi:10.1073/pnas.1305286110

Gebbie L, Benghezal M, Cornillon S, Froquet R, Cherix N, Malbouyres M, Lefkir Y, Grangeasse C, Fache S, Dalous J, Bruckert F, Letourneur F, Cosson P. 2004. Phg2, a kinase involved in adhesion and focal site modeling in Dictyostelium. Mol Biol Cell 15:3915-3925. doi:10.1091/mbc.e03-12-0908

Gerisch G. 2010. Self-organizing actin waves that simulate phagocytic cup structures. Pmc Biophysics 3:7. doi:10.1186/1757-5036-3-7

Gerisch G, Ecke M, Schroth-Diez B, Gerwig S, Engel U, Maddera L, Clarke M. 2009. Selforganizing actin waves as planar phagocytic cup structures. Cell adhesion \& migration 3:373 382. 
994 Gilberti RM, Knecht DA. 2015. Macrophages phagocytose nonopsonized silica particles using a

995 unique microtubule-dependent pathway. Mol Biol Cell 26:518-529. doi:10.1091/mbc.e14-08-

996 1301

997

998

999

1000

1001

1002

1003

1004

1005

1006

1007

1008

1009

1010

1011

1012

1013

1014

1015

1016

1017

Gov NS. 2018. Guided by curvature: shaping cells by coupling curved membrane proteins and cytoskeletal forces. Philosophical Transactions Royal Soc B 373:20170115. doi:10.1098/rstb.2017.0115

Hacker U, Albrecht R, Maniak M. 1997. Fluid-phase uptake by macropinocytosis in Dictyostelium. Journal of Cell Science 110 ( Pt 2):105 112.

Heinrich V, Lee C-Y. 2011. Blurred line between chemotactic chase and phagocytic consumption: an immunophysical single-cell perspective. J Cell Sci 124:3041 3051. doi:10.1242/jcs.086413

Hoeller O, Bolourani P, Clark J, Stephens LR, Hawkins PT, Weiner OD, Weeks G, Kay RR. 2013. Two distinct functions for PI3-kinases in macropinocytosis. J Cell Sci 126:4296-4307. doi:10.1242/jcs. 134015

Hoeller O, Kay RR. 2007. Chemotaxis in the absence of PIP3 gradients. Curr Biol 17:813 817. doi:10.1016/j.cub.2007.04.004

Huang C-H, Tang M, Shi C, Iglesias PA, Devreotes PN. 2013. An excitable signal integrator couples to an idling cytoskeletal oscillator to drive cell migration. Nat Cell Biol 15:1307-1316. doi: $10.1038 / \mathrm{ncb} 2859$

Jasnin M, Beck F, Ecke M, Fukuda Y, Martinez-Sanchez A, Baumeister W, Gerisch G. 2019. The Architecture of Traveling Actin Waves Revealed by Cryo-Electron Tomography. Structure 27:1211-1223.e5. doi:10.1016/j.str.2019.05.009

Jasnin M, Ecke M, Baumeister W, Gerisch G. 2016. Actin Organization in Cells Responding to a Perforated Surface, Revealed by Live Imaging and Cryo-Electron Tomography. Structure 24:1031 1043. doi:10.1016/j.str.2016.05.004 
1018 Jaumouillé V, Cartagena-Rivera AX, Waterman CM. 2019. Coupling of $\beta 2$ integrins to actin by a

1019 mechanosensitive molecular clutch drives complement receptor-mediated phagocytosis. Nat Cell Biol 21:1357-1369. doi:10.1038/s41556-019-0414-2

1021 Jaumouillé V, Waterman CM. 2020. Physical Constraints and Forces Involved in Phagocytosis.

$1022 \quad$ Front Immunol 11:1097. doi:10.3389/fimmu.2020.01097

1023 Kamprad N, Witt H, Schröder M, Kreis CT, Bäumchen O, Janshoff A, Tarantola M. 2018.

1024 Adhesion strategies of Dictyostelium discoideum - a force spectroscopy study. Nanoscale 10:22504-22519. doi:10.1039/c8nr07107a

1026 Katsuno H, Toriyama M, Hosokawa Y, Mizuno K, Ikeda K, Sakumura Y, Inagaki N. 2015. Actin Migration Driven by Directional Assembly and Disassembly of Membrane-Anchored Actin

Kay RR, Williams TD, Manton JD, Traynor D, Paschke P. 2019. Living on soup: macropinocytic Filaments. Cell Reports 12:648-660. doi:10.1016/j.celrep.2015.06.048

1031 Kee Y-S, Ren Y, Dorfman D, Iijima M, Firtel R, Iglesias PA, Robinson DN. 2012. A

1032 mechanosensory system governs myosin II accumulation in dividing cells. Mol Biol Cell

1033 23:1510-1523. doi:10.1091/mbc.e11-07-0601

1034 Kim D-H, Han K, Gupta K, Kwon KW, Suh K-Y, Levchenko A. 2009. Mechanosensitivity of 1035 fibroblast cell shape and movement to anisotropic substratum topography gradients. Biomaterials 30:5433-5444. doi:10.1016/j.biomaterials.2009.06.042

1037 Kortholt A, Bolourani P, Rehmann H, Keizer-Gunnink I, Weeks G, Wittinghofer A, Haastert 1038 PJMV. 2010. A Rap/phosphatidylinositol 3-kinase pathway controls pseudopod formation [corrected]. Mol Biol Cell 21:936 945. doi:10.1091/mbc.e09-03-0177

1040 Kortholt A, Rehmann H, Kae H, Bosgraaf L, Keizer-Gunnink I, Weeks G, Wittinghofer A, Haastert 1041 PJMV. 2006. Characterization of the GbpD-activated Rap1 pathway regulating adhesion and 1042 cell polarity in Dictyostelium discoideum. J Biol Chem 281:23367 23376.

1043 doi:10.1074/jbc.m600804200 
1044 Kowal AS, Chisholm RL. 2011. Uncovering a role for the tail of the Dictyostelium discoideum

1045 SadA protein in cell-substrate adhesion. Eukaryot Cell 10:662-671. doi:10.1128/ec.00221-10

1046 Kwon KW, Park H, Song KH, Choi J-C, Ahn H, Park MJ, Suh K-Y, Doh J. 2012. Nanotopography-

1047 guided migration of T cells. J Immunol 189:2266-2273. doi:10.4049/jimmunol.1102273

1048 Lämmermann T, Bader BL, Monkley SJ, Worbs T, Wedlich-Söldner R, Hirsch K, Keller M, Förster

1049 R, Critchley DR, Fässler R, Sixt M. 2008. Rapid leukocyte migration by integrin-independent

$1050 \quad$ flowing and squeezing. Nature 453:51 55. doi:10.1038/nature06887

1051 Loomis WF, Fuller D, Gutierrez E, Groisman A, Rappel W-J. 2012. Innate non-specific cell

1052 substratum adhesion. Plos One 7:e42033. doi:10.1371/journal.pone.0042033

1053 Maniak M, Rauchenberger R, Albrecht R, Murphy J, Gerisch G. 1995. Coronin involved in

1054 phagocytosis: Dynamics of particle-induced relocalization visualized by a green fluorescent

1055 protein tag. Cell 83:915-924. doi:10.1016/0092-8674(95)90207-4

1056 Marinović M, Mijanović L, Šoštar M, Vizovišek M, Junemann A, Fonović M, Turk B, Weber I, 1057 Faix J, Filić V. 2019. IQGAP-related protein IqgC suppresses Ras signaling during large-scale 1058 endocytosis. Proc National Acad Sci 116:1289 1298. doi:10.1073/pnas.1810268116

1059 Masters TA, Sheetz MP, Gauthier NC. 2016. F-actin waves, actin cortex disassembly and focal 1060 exocytosis driven by actin-phosphoinositide positive feedback. Cytoskeleton 73:180 196.

1061 doi:10.1002/cm.21287

1062 Mathur A, Moore SW, Sheetz MP, Hone J. 2012. The role of feature curvature in contact guidance.

1063 Acta Biomater 8:2595 2601. doi:10.1016/j.actbio.2012.03.025

1064 Miao Y, Bhattacharya S, Edwards M, Cai H, Inoue T, Iglesias PA, Devreotes PN. 2017. Altering

1065 the threshold of an excitable signal transduction network changes cell migratory modes. Nat Cell

1066 Biol 19:329-340. doi:10.1038/ncb3495

1067 Molinie N, Gautreau A. 2018. The Arp2/3 Regulatory System and Its Deregulation in Cancer.

$1068 \quad$ Physiol Rev 98:215 238. doi:10.1152/physrev.00006.2017 
1069 Moores SL, Sabry JH, Spudich JA. 1996. Myosin dynamics in live Dictyostelium cells. Proc National Acad Sci 93:443-446. doi:10.1073/pnas.93.1.443

1071 Mu L, Tu Z, Miao L, Ruan H, Kang N, Hei Y, Chen J, Wei W, Gong F, Wang B, Du Y, Ma G,

1072 Amerein MW, Xia T, Shi Y. 2018. A phosphatidylinositol 4,5-bisphosphate redistribution-based

1073 sensing mechanism initiates a phagocytosis programing. Nat Commun 9:4259.

1074 doi:10.1038/s41467-018-06744-7

Nakajima A, Ishida M, Fujimori T, Wakamoto Y, Sawai S. 2016. The microfluidic lighthouse: an omnidirectional gradient generator. Lab Chip 16:4382-4394. doi:10.1039/c6lc00898d

Nellen W, Silan C, Firtel RA. 1984. DNA-mediated transformation in Dictyostelium discoideum: regulated expression of an actin gene fusion. Mol Cell Biol 4:2890-2898. doi:10.1128/mcb.4.12.2890

Oakley C, Brunette DM. 1993. The sequence of alignment of microtubules, focal contacts and actin filaments in fibroblasts spreading on smooth and grooved titanium substrata. J Cell Sci 106 ( Pt 1):343-54.

Pacheco P, White D, Sulchek T. 2013. Effects of Microparticle Size and Fc Density on Macrophage Phagocytosis. Plos One 8:e60989. doi:10.1371/journal.pone.0060989

Pollard TD. 2007. Regulation of actin filament assembly by Arp2/3 complex and formins. Annu Rev Bioph Biom 36:451 477. doi:10.1146/annurev.biophys.35.040405.101936

1087 Pramanik MK, Iijima M, Iwadate Y, Yumura S. 2009. PTEN is a mechanosensing signal transducer 1088 for myosin II localization in Dictyostelium cells. Genes Cells 14:821-834. doi:10.1111/j.13652443.2009.01312.x

1090 Rajnicek A, Britland S, McCaig C. 1997. Contact guidance of CNS neurites on grooved quartz: 1091 influence of groove dimensions, neuronal age and cell type. Journal of Cell Science 110 ( Pt 1092 23):2905 2913. 
1093 Ray A, Lee O, Win Z, Edwards RM, Alford PW, Kim D-H, Provenzano PP. 2017. Anisotropic

1094

1095

1096

1097

1098

1099

1100

1101

1102

1103

1104

1105

1106

1107

1108

1109

1110

1111 Sasaki AT, Janetopoulos C, Lee S, Charest PG, Takeda K, Sundheimer LW, Meili R, Devreotes

1112

1113

1114

1115

1116

Reichl EM, Ren Y, Morphew MK, Delannoy M, Effler JC, Girard KD, Divi S, Iglesias PA, Kuo SC, Robinson DN. 2008. Interactions between myosin and actin crosslinkers control cytokinesis contractility dynamics and mechanics. Curr Biol 18:471 480. doi:10.1016/j.cub.2008.02.056

Reig G, Pulgar E, Concha ML. 2014. Cell migration: from tissue culture to embryos. Development 141:1999-2013. doi:10.1242/dev.101451

Reversat A, Gaertner F, Merrin J, Stopp J, Tasciyan S, Aguilera J, Vries I de, Hauschild R, Hons M, Piel M, Callan-Jones A, Voituriez R, Sixt M. 2020. Cellular locomotion using environmental topography. Nature 582:582-585. doi:10.1038/s41586-020-2283-z

Rougerie P, Miskolci V, Cox D. 2013. Generation of membrane structures during phagocytosis and chemotaxis of macrophages: role and regulation of the actin cytoskeleton. Immunol Rev 256:222 239. doi:10.1111/imr.12118

Saito N, Sawai S. 2020. Three-dimensional morphodynamics simulations of macropinocytic cups. Biorxiv 2020.06.22.165027. doi:10.1101/2020.06.22.165027

Sales A, Holle AW, Kemkemer R. 2017. Initial contact guidance during cell spreading is contractility-independent. Soft Matter 13:5158-5167. doi:10.1039/c6sm02685k

PN, Firtel RA. 2007. G protein-independent Ras/PI3K/F-actin circuit regulates basic cell motility. J Cell Biology 178:185 191. doi:10.1083/jcb.200611138

Schroth-Diez B, Gerwig S, Ecke M, Hegerl R, Diez S, Gerisch G. 2009. Propagating waves separate two states of actin organization in living cells. Hfsp J 3:412-427.

doi:10.2976/1.3239407 
1117 Sharma VP, Beaty BT, Patsialou A, Liu H, Clarke M, Cox D, Condeelis JS, Eddy RJ. 2012.

1118 Reconstitution of in vivo macrophage-tumor cell pairing and streaming motility on one-

1119 dimensional micro-patterned substrates. Intravital 1:77-85. doi:10.4161/intv.22054

1120 Sun X, Driscoll MK, Guven C, Das S, Parent CA, Fourkas JT, Losert W. 2015. Asymmetric

1121 nanotopography biases cytoskeletal dynamics and promotes unidirectional cell guidance. Proc

1122 National Acad Sci 112:12557-12562. doi:10.1073/pnas.1502970112

1123 Swanson JA. 2008. Shaping cups into phagosomes and macropinosomes. Nat Rev Mol Cell Bio

$1124 \quad 9: 639$ 649. doi:10.1038/nrm2447

1125 Swanson JA, Baer SC. 1995. Phagocytosis by zippers and triggers. Trends Cell Biol 5:89-93.

1126 doi:10.1016/s0962-8924(00)88956-4

1127 Taniguchi D, Ishihara S, Oonuki T, Honda-Kitahara M, Kaneko K, Sawai S. 2013. Phase

1128 geometries of two-dimensional excitable waves govern self-organized morphodynamics of

1129 amoeboid cells. Proc National Acad Sci 110:5016-5021. doi:10.1073/pnas.1218025110

1130 Teixeira AI, McKie GA, Foley JD, Bertics PJ, Nealey PF, Murphy CJ. 2006. The effect of

1131 environmental factors on the response of human corneal epithelial cells to nanoscale substrate

1132 topography. Biomaterials 27:3945-3954. doi:10.1016/j.biomaterials.2006.01.044

1133 Tsujioka M, Yumura S, Inouye K, Patel H, Ueda M, Yonemura S. 2012. Talin couples the

1134 actomyosin cortex to the plasma membrane during rear retraction and cytokinesis. Proc National

1135 Acad Sci 109:12992 12997. doi:10.1073/pnas.1208296109

1136 Tunnacliffe E, Corrigan AM, Chubb JR. 2018. Promoter-mediated diversification of transcriptional

1137 bursting dynamics following gene duplication. Proc National Acad Sci 115:201800943.

1138 doi:10.1073/pnas. 1800943115

1139 Veltman DM, Williams TD, Bloomfield G, Chen B-C, Betzig E, Insall RH, Kay RR, Swanson J.

1140 2016. A plasma membrane template for macropinocytic cups. Elife 5:e20085.

1141 doi:10.7554/elife.20085 
1142 Weiner OD, Marganski WA, Wu LF, Altschuler SJ, Kirschner MW. 2007. An actin-based wave

1143 generator organizes cell motility. Plos Biol 5:e221. doi:10.1371/journal.pbio.0050221

1144 Wilkinson PC, Shields JM, Haston WS. 1982. Contact guidance of human neutrophil leukocytes.

1145 Exp Cell Res 140:55-62. doi:10.1016/0014-4827(82)90155-0

1146 Williams TD, Kay RR. 2018. The physiological regulation of macropinocytosis during

1147 Dictyostelium growth and development. J Cell Sci 131:jcs213736. doi:10.1242/jcs.213736

1148 Wójciak-Stothard B, Curtis A, Monaghan W, Macdonald K, Wilkinson C. 1996. Guidance and

1149 Activation of Murine Macrophages by Nanometric Scale Topography. Exp Cell Res 223:426-

$1150 \quad$ 435. doi:10.1006/excr.1996.0098

1151 Wu Z, Su M, Tong C, Wu M, Liu J. 2018. Membrane shape-mediated wave propagation of cortical 1152 protein dynamics. Nat Commun 9:136. doi:10.1038/s41467-017-02469-1

1153 Yang Y, Xiong D, Pipathsouk A, Weiner OD, Wu M. 2017. Clathrin Assembly Defines the Onset

1154 and Geometry of Cortical Patterning. Dev Cell 43:507-521.e4. doi:10.1016/j.devcel.2017.10.028

1155 Yoshida M, Stadler J, Bertholdt G, Gerisch G. 1984. Wheat germ agglutinin binds to the contact

1156 site A glycoprotein of Dictyostelium discoideum and inhibits EDTA-stable cell adhesion. The

1157 EMBO Journal 3:26632670.

1158 Zhao W, Hanson L, Lou H-Y, Akamatsu M, Chowdary PD, Santoro F, Marks JR, Grassart A,

1159 Drubin DG, Cui Y, Cui B. 2017. Nanoscale manipulation of membrane curvature for probing

1160 endocytosis in live cells. Nat Nanotechnol 12:750-756. doi:10.1038/nnano.2017.98 